ARTICLE

\title{
Cut-like homeobox 1 (CUX1) tumor suppressor gene haploinsufficiency induces apoptosis evasion to sustain myeloid leukemia
}

Emmanuelle Supper ${ }^{1,4}$, Saskia Rudat ${ }^{1,4}$, Vivek Iyer ${ }^{1}$, Alastair Droop ${ }^{1}$, Kim Wong $\mathbb{B}^{1}$, Jean-François Spinella ${ }^{2}$, Patrick Thomas', Guy Sauvageau (1) ${ }^{2}$, David J. Adams (i) ${ }^{1} \&$ Chi C. Wong (1) ${ }^{1,3 凶}$

While oncogenes promote tumorigenesis, they also induce deleterious cellular stresses, such as apoptosis, that cancer cells must combat by coopting adaptive responses. Whether tumor suppressor gene haploinsufficiency leads to such phenomena and their mechanistic basis is unclear. Here, we demonstrate that elevated levels of the anti-apoptotic factor, CASP8 and FADD-like apoptosis regulator (CFLAR), promotes apoptosis evasion in acute myeloid leukemia (AML) cells haploinsufficient for the cut-like homeobox 1 (CUX1) transcription factor, whose loss is associated with dismal clinical prognosis. Genome-wide CRISPR/ Cas9 screening identifies CFLAR as a selective, acquired vulnerability in CUX1-deficient AML, which can be mimicked therapeutically using inhibitor of apoptosis (IAP) antagonists in murine and human AML cells. Mechanistically, CUX1 deficiency directly alleviates CUX1 repression of the CFLAR promoter to drive CFLAR expression and leukemia survival. These data establish how haploinsufficiency of a tumor suppressor is sufficient to induce advantageous anti-apoptosis cell survival pathways and concurrently nominate CFLAR as potential therapeutic target in these poor-prognosis leukemias.

\footnotetext{
${ }^{1}$ Experimental Cancer Genetics, Wellcome Sanger Institute, Hinxton, UK. ${ }^{2}$ The Leucegene Project at Institute for Research in Immunology and Cancer, Université de Montréal, 2950 Chemin de Polytechnique Pavillon, Marcelle-Coutu, Montréal, QC, Canada. ${ }^{3}$ Department of Haematology, Addenbrooke's Hospital, Cambridge, UK. ${ }^{4}$ These authors contributed equally: Emmanuelle Supper and Saskia Rudat. ${ }^{\circledR}$ email: cw6@sanger.ac.uk
} 
C ancers arise following the accumulation of somatic genetic lesions which can activate oncogenes or disable tumor suppressors. While biallelic inactivation of tumor suppressor genes is a well-established mechanism underpinning tumorigenesis ${ }^{1}$, the contribution of tumor suppressor gene haploinsufficiency in tumor development is less well characterized. In this scenario, monoallelic loss of the tumor suppressor is sufficient to promote tumor development. The human genome harbors around 1,400 putative transcription factor genes ${ }^{2}$ of which $\sim 100$ genes have been found to be mutated in cancer and approximately half of these genes are implicated as tumor suppressors $^{3}$, with only a handful of these genes implicated as haploinsufficient tumor suppressors ${ }^{4}$. Although some progress has been made in the therapeutic targeting of gain-of-function oncogenic transcription factors ${ }^{5}$, loss-of-function transcription factor tumor suppressors represent difficult targets for direct therapeutic intervention, necessitating alternative approaches to target malignancies harboring such lesions. It is recognized that cancer cells can develop critical dependencies on cellular pathways that abrogate cancer-induced cellular stresses-a phenomenon known as non-oncogene addiction ${ }^{6,7}$. For instance, KRAS, $B R A F$ and $M Y C$ oncogenes can induce proteotoxic, DNA replication, mitotic, metabolic and oxidative stresses, that must be countered within cancer cells for their survival ${ }^{8-14}$. While targeting these buffering pathways represents an attractive, alternative strategy to treat cancers driven by oncogenes, it remains unclear whether tumor suppressor genes-and in particular haploinsufficiency of these genes-can engender cells with similar vulnerabilities that can be exploited therapeutically. Moreover, the identification of these actionable cellular dependencies and the molecular mechanisms underlying them remains challenging.

Here, we focussed on the chromosome $7 \mathrm{q}$ homeodomain transcription factor CUX1 (also known as CUTL1 or CDP) which is targeted by monoallelic chromosome 7 deletions [-7/del $(7 q)]$ and somatic, heterozygous, truncating mutations in diverse myeloid malignancies such as myelodysplasia (MDS) and myelodysplasia/myeloproliferative neoplasms (MDS/MPN) - both of which can progress to $\mathrm{AML}^{15-19}$. CUX1 mutations are more common in myelomonocytic diseases such as chronic myelomonocytic leukemia (CMML; an MDS/MPN characterized by monocytosis and hematopoietic dysplasia) ${ }^{18,20}$ and high-risk $\operatorname{MDS}^{19}$, with biallelic CUX1 mutations being infrequent, consistent with CUX1 being predominantly a haploinsufficient tumor suppressor ${ }^{18,21}$. CUX1 harbors four DNA-binding motifs, including three CUT repeats and a homeodomain. While full-length CUX1 (CUX1 $\left.{ }^{\mathrm{p} 200}\right)$ acts as a transcriptional repressor, proteolytic cleavage generates a Cterminal CUX1 110 isoform with transcriptionally activating or repressive properties depending on promoter context ${ }^{22}$. Our previous work in mice identified Cuxl as a potential T-cell leukemia tumor suppressor gene, with Cuxl-deficient tumors exhibiting increased PI3K signaling, due to loss of CUX1-regulated expression of the gene encoding phosphoinositide 3-kinase interacting protein 1 (PIK3IP1)-a PI3K inhibitor ${ }^{18,23}$. However, the role of CUX1 loss and PIK3IP1 in myeloid malignancies remains obscure. Knockdown of Cux1 in mice to levels approximating Cux1 haploinsufficiency led to an MDS-like disease, but AML was not apparent, suggesting the need for cooperating genetic lesions ${ }^{24}$. In this regard, genomic studies of CUX1-mutated and $-7 / \operatorname{del}(7 q)$ myeloid malignancies have uncovered cooccurring inactivating mutations in other myeloid malignancyassociated genes such as TET2 and ASXL1 in addition to gain-offunction mutations in FLT3 and the RAS pathway, but their causal role in CUX1-deficient AML development is unclear ${ }^{19,25-27}$. Despite the dismal prognosis conferred by both $-7 /($ del7q) lesions and heterozygous, inactivating CUX1 mutations in myeloid malignancies $^{17-19}$, which has led to the incorporation of $-7 /$ del (7q) lesions in international clinical prognostic scoring systems ${ }^{15,16}$, there are no specific therapies for the treatment of CUX1-deficient myeloid malignancies.

Apoptosis evasion is acknowledged to be critical for the development and sustained growth of tumors and is divided into extrinsic and intrinsic (mitochondrial) pathways ${ }^{28,29}$. CFLAR (also known as $C F L I P$ ), encodes three main protein isoforms (cCFLIP $_{L}, c-$ FLIP $_{S}$ and $c-$ FLIP $_{R}$ ) which can act as antagonists of death receptor-mediated extrinsic apoptosis by competing with caspase- 8 during recruitment to ligand-activated receptors of the TNF family, thereby curtailing caspase- 8 processing and activation $^{30}$. In addition, CFLAR isoforms can modulate cell death via necroptosis: a programmed form of necrosis mediated by receptor-interacting protein kinase (RIPK) and mixed lineage kinase domain-like pseudokinase (MLKL) proteins. RIPK1 is known to phosphorylate and activate RIPK3, which in turn phosphorylates the key necroptosis effector, MLKL, leading to protein oligomerization and translocation to the plasma membrane ${ }^{31-36}$. In this study, we sought to identify genetic vulnerabilities in CUX1-deficient AML cells using CRISPR/ Cas9 screening to assess whether CUX1 deficiency invokes adaptive cellular pathways critical for leukemia survival. Using a murine Cux1-deficient leukemia model and human cells, we establish that CUX1-deficient leukemia cells rely on elevated levels of CFLAR to evade apoptosis. Mechanistically, we show that CUX1 haploinsufficiency alleviates CUX1-mediated transcriptional repression of CFLAR expression to abrogate cellular apoptosis. Our study illuminates the molecular pathogenesis of CUX1-deficient leukemias and establishes that tumor suppressor gene haploinsufficiency can provoke collateral addiction to adaptive cellular pathways of potential therapeutic importance.

\section{Results}

CRISPR/Cas9 screening identifies CFLAR as a selective vulnerability in $\boldsymbol{C U X 1 - d e f i c i e n t ~ c e l l s . ~ I n ~ a n ~ i n i t i a l ~ a p p r o a c h ~ t o ~}$ determine whether tumor suppressor loss could induce adaptive cellular responses vital for survival, we performed genome-wide CRISPR/Cas9 drop-out screens in isogenic myeloid cancer cells that differed solely in CUX1 status (Fig. 1a). We selected U937 cells since they have a myelomonocytic phenotype in addition to being deficient in $T E T 2^{37}$; these characteristics are enriched in CUX1-mutated myeloid malignancies ${ }^{18,19}$. Furthermore, they represent one of the few myelomonocytic AML lines without preexisting chromosome 7 copy number alterations, rendering them a feasible background for our approach. Three clonal CUX1-/and three CUX1-wild-type U937 cell lines were generated by transiently transfecting cells with plasmids engineered to express single guide RNAs (sgRNAs) targeting CUX1 exon 18, Cas9 endonuclease and EGFP. Single $\mathrm{EGFP}^{+}$clones were expanded in culture for genotyping and immunoblotting to confirm CUX1 disruption (Fig. 1b, c). Loss of CUX1 expression did not affect proliferation, cell cycle distribution, immunophenotype or steadystate apoptosis of the cells in vitro compared with wild-type cells (Supplementary Fig. 1a-d).

Each cell line was infected with lentiviral supernatants to express comparable functional levels of Cas9 endonuclease (Supplementary Fig. 1e, f). CRISPR/Cas9 drop-out screening was performed using an sgRNA library containing 90,709 sgRNAs targeting 18,010 genes at 200-fold coverage (see Methods). Whereas sgRNAs targeting known essential genes were depleted from both experimental groups as expected, the abundance of the majority of sgRNAs, including control sgRNAs, were not preferentially altered in $C U X 1^{-/-}$cells compared with wildtype after 21 days from sgRNA library infection (Fig. 1d and Supplementary Data 1). Remarkably, using a Z score threshold 
a

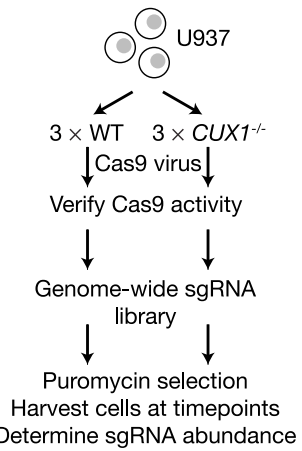

b

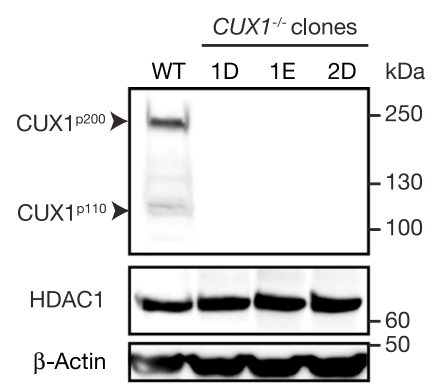

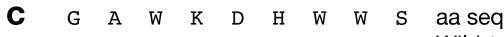
GGTGCCTGGAAGGACCACTGGTGGAGC Wild-type

GGTGCCCGG----------GTGGAGC Clone 1D GGTGCCTGGAAGGACCACT-GTGGAGC

GGTGCCTGGAAGGACCACT-GTGGAGC Clone 1E GGTGCCTGGAAGGACCACT----GAGC

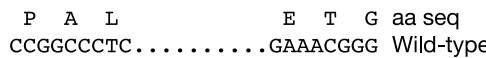

CCGGCCC--180 bp inv-AAACGGG Clone 2D CCGGCCC--180 bp inv-AAACGGG d

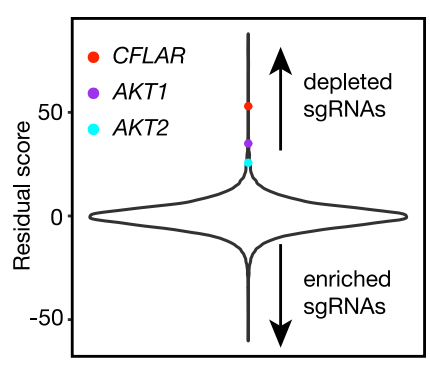

f
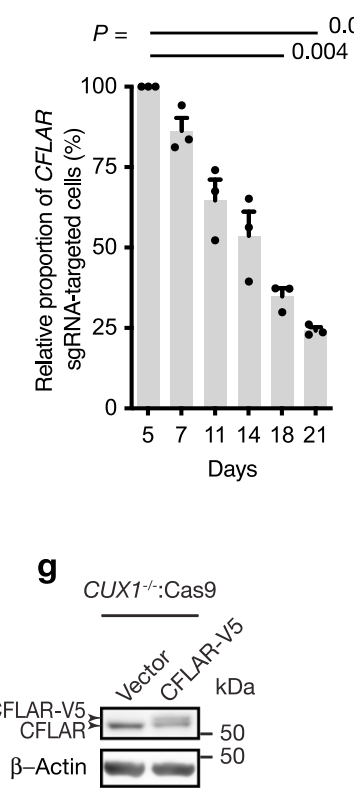

- Vector

- CFLAR-V5

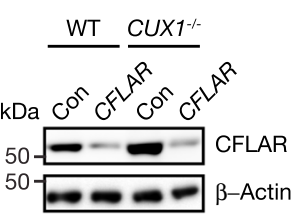

CFLAR

e

positive regulation of PUD protein catabolic process positive regulation of histone acetylation

protein sumoylation protein phosphorylationexecution phase of apoptosispositive regulation of transcription from RNA polymerase II promoterregulation of transcription from RNA polymerase III promoterDNA replication cellular response to DNA damage stimulus positive regulation of transcription, DNA-templatednegative regulation of transcription, DNA-templatedmitotic nuclear divisioncovalent chromatin modificationregulation of transcription from RNA polymerase II promotertranscription, DNA-templatedregulation of signal transduction by $\mathrm{p} 53$ class mediatorsister chromatid cohesionchromatin remodeling cell division histone $\mathrm{H} 3$ acetylation

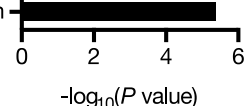

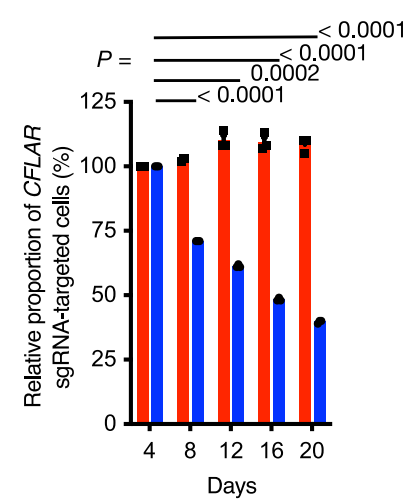

CUX 1 -

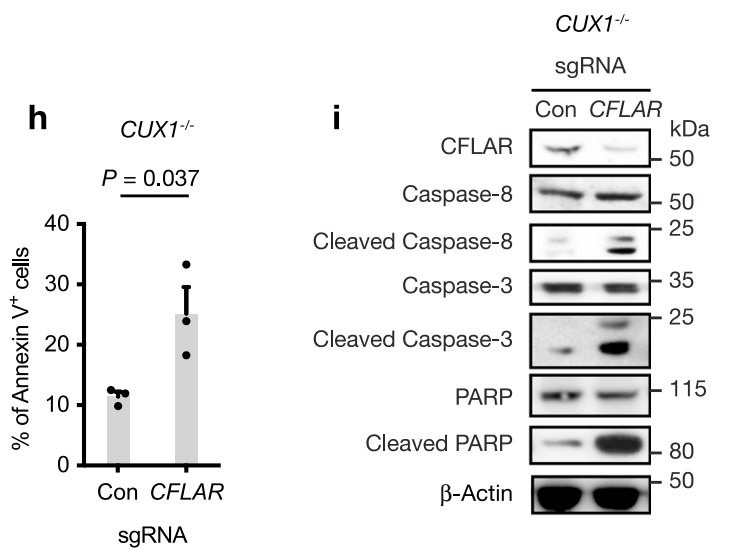

Gene ontology analysis implicated a number of additional cellular processes which were important for cell survival in CUX1-deficient cells, including genes involved in apoptosis regulation (Fig. 1e and Supplementary Data 2). Notably, previous work has shown that Fas-deficient mice display expanded myeloid progenitor numbers and concurrent expression of BCL2 in a Fas-deficient background can precipitate AML in
$>3.5$, we identified 98 selective dependency genes in CUX1 ${ }^{-1-}$ cells including both $A K T 1$ and $A K T 2$, with respective ranks at positions 24 and 96 in our screen ( $\mathrm{Z}$ score; $A K T 1=4.81$, $A K T 2=3.53$ ), suggesting that CUX1-deficient AML cells could also be more dependent on PI3K signaling consistent with our previously reported sensitivity of CUX1-deficient T-cell leukemias to PI3K inhibition ${ }^{18}$ (Fig. 1d). 
Fig. 1 CRISPR/Cas9 drop-out screening identifies genetic vulnerabilities in CUX1-deficient cells. a Scheme of CRISPR/Cas9 drop-out screen to identify selective vulnerabilities in CUX1-/- cells. WT, wild-type. $\mathbf{b}$ Immunoblot showing loss of CUX1 protein expression in three CUX1-/- clones compared with wild-type (WT). HDAC1 and $\beta$-Actin were used as loading controls. The experiment was done twice with similar results. $\mathbf{c}$ Partial sequence of $C U X 1$ exon 18 from CUX1-/- clones 1D, 1E and 2D showing wild-type genomic and amino acid (aa) sequences (top) and clone-specific mutated sequences (bottom); inv, inversion. $\mathbf{d}$ Distribution of residual scores as a measure of gene essentiality in CUX1-/- cells compared with wild-type cells. Positions of CFLAR, AKT1 and AKT2 genes are highlighted. Target genes with higher residual scores exhibit higher degree of potential synthetic lethality in $C U X 1^{-/-}$than in wild-type cells. e Functional annotation of dependency genes in CUX1-/- cells identified by CRISPR/Cas9 screening. PUD, proteasomal ubiquitin-dependent. $P$ values were not adjusted for multiple comparisons. Apoptosis pathway is highlighted in red. f Competitive sgRNA assay (left) showing preferential loss of CFLAR sgRNA-targeted cells in CUX1-/- U937 cells compared with wild-type. The results from CUX1-/- U937 cells were normalized to wild-type cells at each time point. Day 5 was designated a starting value of $100 \%$ and subsequent values were calculated relative to day 5 . Immunoblot (right) confirming depletion of target protein by CFLAR-targeting sgRNA compared with control sgRNA (Con) in wild-type and CUX1-/- cells. The experiment was done three times. $\mathbf{g}$ Immunoblot showing expression of CFLAR-V5 in CUX1-/-:Cas9 cells (left) and rescue of lethality following CFLAR-targeting sgRNA infection in CFLAR-V5-expressing cells (right). Empty vector was used as control (vector). Results were normalized to day 4 in each case. Statistically significant comparisons shown are between empty vector-expressing groups. The experiment was done three times. $\mathbf{h}$ Percentage of Annexin- $\mathrm{V}^{+}$cells at day 5 following CFLAR-targeting sgRNA infection compared with control sgRNA (Con) in CUX1-/- cells. The experiment was done three times. $\mathbf{i}$ Immunoblot of indicated proteins at day 5 following CFLAR-targeting sgRNA infection compared with control sgRNA (Con) in CUX1-/- cells. The experiment was done twice with similar results. CUX1-/- clone 1D cell line was used in $\mathbf{f - i}$. One-way ANOVA with Dunnett's test for multiple comparisons and repeated measures (f, $\mathbf{g})$, Two-tailed, unpaired $t$-test $(\mathbf{h})$.

double-mutant mice ${ }^{38}$, implying that defective apoptosis control could be important in AML pathogenesis. Among the top-ranked genes, CFLAR was the most highly positioned apoptosisregulating gene in our screen (fifth ranked, $\mathrm{Z}$ score $=7.28$; Fig. 1d), leading us to focus on CFLAR as a specific vulnerability in CUX1-deficient cells. Interestingly and in contrast, sgRNAs targeting pro-apoptotic genes such as BCL2L11 (also known as $B I M), B C L 2 L 15$ and BCL7B were enriched in the CUX1-deficient cells, suggesting that apoptosis attenuation promoted survival of CUX1-deficient cells compared with wild-type. To verify our screening results, we performed competitive sgRNA depletion assays using individual CFLAR-targeting or control sgRNAs linked to BFP in two $C U X 1^{-/-}$and wild-type cell line clones. Expression of CFLAR-targeting sgRNA in CUX1-/- or wild-type cells led to greater depletion of targeted cells in CUX1-lcompared with wild-type backgrounds, while expression of control sgRNA had no significant effect (Fig. If and Supplementary Fig. $1 \mathrm{~g}-\mathrm{i}$ ). We noted that cells remaining at 21 days following infection with CFLAR-targeting sgRNAs escaped CFLAR depletion, which likely accounts for their survival at this time (Supplementary Fig. 1j). Additionally, ectopic expression of a CRISPR/Cas9 cleavage-resistant CFLAR isoform in CUX1-/cells, abolished the negative impact of CFLAR-targeting sgRNA expression on proliferation, excluding off-target effects from the CFLAR-targeting sgRNA as the cause of our observed phenotype (Fig. 1g).

We next sought to investigate the basis of CFLAR dependency in CUX1-deficient cells. We noted that only CFLAR isoforms corresponding to the long $\mathrm{c}-\mathrm{CFLIP} \mathrm{L}_{\mathrm{L}}$ protein variant were detected in U937 cells. Since this CFLAR isoform has been ascribed both anti- and pro-apoptotic functions ${ }^{39}$, we evaluated the impact of CFLAR depletion in CUX1-/- cells. We found that CFLAR depletion led to an increase in Annexin- $\mathrm{V}^{+}$apoptotic cells (Fig. 1h) and cleaved forms of caspase-3, caspase-8 and PARP indicative of activation of the apoptosis-signaling cascade (Fig. 1i), suggesting that CFLAR acts to restrain apoptosis. To exclude the possibility that CFLAR dependency was unique to CUX1-1U937 cells, we reproduced the selective impact of CFLAR depletion in engineered $C U X 1^{-1-}$ and wild-type THP-1 myeloid cancer cells, which also possess two wild-type CUX1 alleles (Supplementary Fig. 1k-m). Furthermore, since CUX1 haploinsufficiency predominates over complete CUX1 loss in myeloid malignancies, we wished to establish the relevance of findings by comparing the impact of CFLAR depletion in CUX1-haploinsufficient cells. To model CUX1 haploinsufficiency in U937 and
THP-1 cells, we used an shRNA (shCUX1) approach to stably deplete CUX1 to approximately 50\% of wild-type levels by selecting for puromycin-resistant cells (Supplementary Fig. 1n). Non-targeting shRNAs (shCon) were used as controls. These cells were transduced with lentiviral supernatants to express Cas 9 endonuclease followed by CFLAR-targeting sgRNAs linked to BFP. Over time, cells harboring CFLAR-targeting sgRNAs as marked by BFP were preferentially depleted in shCUX1 cells compared with shCon cells, supporting the idea that CFLAR is a specific vulnerability in both CUX1-haploinsufficent and CUX1knockout cells (Supplementary Fig. 1o, p).

Cux1 haploinsufficiency does not lead to leukemia. In order to investigate whether CUX1-deficient primary leukemic cells are more reliant on CFLAR, we sought to generate a CUX1-deficient leukemia model. To this end, we generated conditional

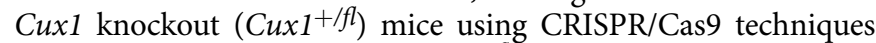
(Supplementary Fig. 2a-c). Cux $1^{+f l}$ mice were crossed to the Vav-iCre recombinase strain, in which Cre recombinase expression is driven by the Vav1 promoter that is active in hematopoietic cells. Efficient Cuxl deletion in $\mathrm{Vav}_{-} i \mathrm{Cre}^{+}$animals was confirmed by PCR (Supplementary Fig. 2d) and CUX1 protein expression was lost in a Cux $1^{f l}$ allele-dependent manner without detectable truncated N-terminal CUX1 isoforms (Supplementary Fig. 2e).

At six and twelve months, homozygous Cuxl deletion in $\mathrm{CuxI}^{f l / f l} ; \mathrm{Vav}^{-i \mathrm{Cre}^{+}}$mice led to progressive macrocytic anemia and leukocytosis due to monocytosis and neutrophilia, which was similarly observed in Cuxl-knockdown mice ${ }^{24}$ (Supplementary Fig. 3a-e). While a subset of aged Cuxiflffl:Vav-iCre ${ }^{+}$mice eventually developed MDS/MPN resembling human CMML starting around one year of age, characterized by variable splenomegaly, hematopoietic dysplasia and organ infiltration with myelomonocytic cells (Supplementary Fig. $3 \mathrm{f}-\mathrm{h}$ ), no cases of acute leukemia were observed. In contrast, haploinsufficient $\mathrm{Cux1}^{+/ f l} ; \mathrm{Vav}$-iCre $^{+}$(referred to as $\mathrm{Cux1^{+/- }}$ ) mice displayed mild macrocytic anemia without significant abnormalities in absolute leukocyte numbers (Supplementary Fig. 3k-m), although myelodysplastic features were evident in blood and bone marrow cytospins (Supplementary Fig. 3f-j). These observations suggested that additional mutations were required for the development of myeloid malignancies such as MDS/MPN and AML that are also associated with CUX1 haploinsufficiency in humans. Interestingly, platelet counts were elevated in $\mathrm{Cux} \mathrm{I}^{\mathrm{fl} / \mathrm{f}} ; \mathrm{Vav}-\mathrm{iCr} e^{+}$

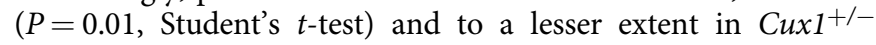


( $P=0.08$, Student's $t$-test) mice at 12 months of age (Supplementary Fig. $3 n$ ), which was associated with increased numbers of megakaryocytes in bone marrow sections compared with control mice, suggesting a role for Cuxl in megakaryopoiesis (Supplementary Fig. 3o).

Cux1 haploinsufficiency cooperates with Flt3 ${ }^{I T D}$ mutation leading to CMML and AML in vivo. Since we wished to establish a CUX1-haploinsufficient AML model, we searched for mutations which cooccur with $-7 / \operatorname{del}(7 \mathrm{q})$ lesions in human AML. As activating FMS-like tyrosine kinase 3 (FLT3) mutations are common $(\sim 30 \%)$ in myeloid malignancies with normal karyotype and are found in $\sim 10 \%$ cases with $-7 / \operatorname{del}(7 q)$ lesions ${ }^{26,27,40,41}$, we generated $\mathrm{Cux} 1^{+/ f l} ; \mathrm{Flt}^{I T D /+} ; \mathrm{Vav}$-iCre ${ }^{+}$(herein referred to as Cux $1^{+/-} ;$Flt $3^{I T D}$ ) mice to model Cux1 haploinsufficiency in the context of a heterozygous, activating Flt $3^{I T D}$ mutation-which alone leads to chronic myeloproliferative disease but not AML in knock-in mice ${ }^{40,42}$. Strikingly, all $\mathrm{Cu} 1^{+/-}$;Flt3 ${ }^{\text {ITD }}$ mice developed lethal hematologic disease with a median survival of 28 weeks, while control (Con) and single-allele (Cux1 $1^{+-}$and Flt $3^{I T D}$ ) groups remained healthy (Fig. 2a). Blood from 10-weekold Cux $1^{+-} ;$Flt $3^{I T D}$ mice revealed macrocytic anemia (Fig. 2b, c), neutrophilia and monocytosis (Fig. 2d, e). Blood smears confirmed leukocytosis with dysplastic monocytes and neutrophils (Fig. 2f). In addition, splenomegaly was evident in Cux $1^{+/-}$;Flt3 ITD mice (Fig. 2g). Surprisingly, while pathologic

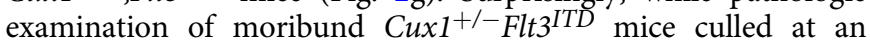
earlier age revealed infiltration of liver, spleen and bone marrow with excessive numbers of myelomonocytic cells leading to effacement of normal tissue architecture compatible with a diagnosis of CMML (Fig. 2h-k; square symbols Fig. 2a), older animals succumbed to AML following on from prior CMML (circle symbols Fig. 2a). In CMML cases, increased mature myeloid cell numbers were evident by flow cytometry (Fig. 2l) and myeloperoxidase immunohistochemistry (Fig. 2m), while AML blasts were c-Kit ${ }^{+}$(Fig. 2n, o).

To assess whether leukemias were transplantable, we injected $2 \times 10^{5}$ bone marrow cells from two Cux $1^{+/-} ; F l t 3^{I T D}$ mice diagnosed with AML or Flt3 ${ }^{I T D}$ mice along with the same number of wild-type bone marrow support cells into irradiated recipient wild-type mice. While mice injected with Flt $3^{I T D}$ cells remained healthy during this study, recipients of $\mathrm{Cux} 1^{+/-} ; \mathrm{Flt3} 3^{I T D}$ cells developed pallor and cachexia which compromised their survival (Fig. 3a). Splenic enlargement and gross pallor of femurs

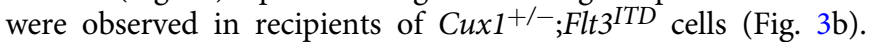
Blood count analysis at necropsy revealed anemia and leukocytosis with the latter attributable to increased neutrophils, monocyte and blasts in mice transplanted with $\mathrm{Cux} 1^{+/-} ;$Flt $3^{I T D}$ cells, but not Flt3 ${ }^{I T D}$ cells (Fig. 3c). Blood smears from these animals demonstrated numerous hypolobated, dysplastic neutrophils (Fig. 3d). Histopathology showed that bone marrow, spleen and liver tissues were infiltrated with myelomonocytic and immature blast cells as observed in the peripheral blood (Fig. $3 \mathrm{e}-\mathrm{g}$ ) and bone marrow cytospins demonstrated numerous blast cells along with more mature myelomonocytic cells (Fig. 3h). Flow cytometry of blood, bone marrow and spleen revealed an increase in cells expressing Gr1 and Mac1 consistent with myeloid expansion (Fig. 3i). We also genotyped primary

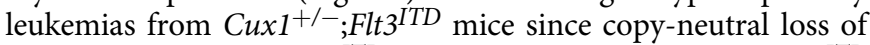
heterozygosity at the Flt $3^{I T D}$ locus leading to doubling of Flt $3^{I T D}$ gene dosage has been associated with disease progression in murine and human $F L T 3^{I T D}$-associated $\mathrm{AML}^{43,44}$, but we found no evidence of this phenomenon at either Flt3 $3^{I T D}$ or Cux 1 loci (Fig. 3j), implying that heterozygosity for both alleles was sufficient to promote leukemogenesis. These data indicate that
Cux1 haploinsufficiency is sufficient to promote transplantable AML in concert with the Flt3 ${ }^{I T D}$ allele.

Cux1 haploinsufficiency affects hematopoietic stem and progenitor cell development in $\mathrm{Cux1}^{+/-} ; \mathrm{Flt3}^{I T D}$ mice. Since Flt $3^{I T D}$ expression in mice leads to abnormal hematopoietic stem cell (HSC) and myeloid progenitor (MP) differentiation ${ }^{40}$, we determined the frequency of HSCs, MPs and their subsets using flow cytometry in 10-12-week-old control (Con), Cux $1^{+/-}$, Flt3 ${ }^{I T D}$ and Cux $1^{+/-} ;$Flt3 ${ }^{I T D}$ mice. Bone marrow cellularity was increased in $\mathrm{Cux} 1^{+/-} ;$Flt3 ${ }^{I T D}$ and, to a lesser extent, in Flt3 ${ }^{I T D}$ mice (Fig. 4a). Absolute numbers of LSK $\left(\mathrm{Lin}^{-} \mathrm{Sca}-1^{+} \mathrm{c}-\mathrm{Kit}^{+}\right.$) HSCs were increased in $\mathrm{Cu} \times 1^{+/-} ; \mathrm{Flt} 3^{I T D}$ mice, but not significantly increased in other groups at this age (Fig. 4b). Using CD150 and CD48 signaling lymphocytic activation molecule (SLAM) markers, we characterized the LSK compartment further to define phenotypic long-term HSC (LT-HSC), short-term HSC (ST-HSC) and multipotent progenitor (MPP) cells ${ }^{45}$. Strikingly, the increase in LSK cells in Cux1 $1^{+/-} ;$Flt3 ${ }^{I T D}$ mice was mainly attributable to expansion of the MPP population at the expense of LT-HSC and ST-HSC populations compared with the control group (Fig. 4c-e). Although LT-HSC frequencies are known to be reduced in Flt3 ITD mice ${ }^{42}$, this reduction was even more pro-

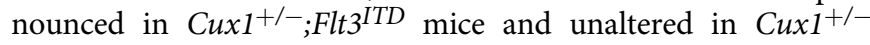
mice compared with controls (Fig. 4c). Assessment of LK ( $\mathrm{Lin}^{-}$

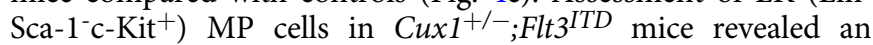
expansion of absolute numbers of LK MP cells (Fig. 4f), which was mainly due to increased numbers of granulocyte-monocyte progenitors (GMPs) rather than common myeloid progenitors (CMPs), whereas the frequency of megakaryocyte-erythroid progenitors (MEPs) was decreased compared with other groups (Fig. $4 \mathrm{~g}-\mathrm{i}$ ). Bone marrow lineage analysis revealed an increase in the proportion of $\mathrm{Gr}-1^{+} \mathrm{Mac1}^{+}$myeloid cells in Flt3 ${ }^{I T D}$ and Cux $1^{+/-}$;Flt3 $3^{I T D}$ mice compared with other groups (Fig. 4j). Taken together, these data show that Cuxl haploinsufficiency and Flt $3^{I T D}$ cooperate to alter hematopoietic stem and progenitor cell homeostasis to a greater extent than each mutant allele alone. The changes in GMP and MEP numbers provide potential explanations for anemia and dominant myelopoiesis observed in Cux $1^{+/-} ;$Flt3 ${ }^{I T D}$ mice.

Apoptosis defects in $\mathrm{Cux1}^{+/-}$;Flt3 ${ }^{I T D}$ mice. Previous analysis of Flt $3^{I T D}$ mice has shown that Flt $3^{I T D}$ expression is associated with reduced apoptosis of both LSK HSCs and LK myeloid progenitors in a dose-dependent manner ${ }^{40}$. To assess the apoptosis status of Cux $1^{+/-} ;$Flt $3^{I T D}$ cells compared with other groups, we performed flow cytometry for Annexin-V (AV) in LSK and LK cell compartments using 10-12-week-old mice. A fixable viability dye (FVD) was used to detect dead cells. Cux1 $1^{+/-}$and $\mathrm{Cux1^{+/- }}$; Flt3 ${ }^{I T D}$ LSK HSCs exhibited a reduced frequency of early apoptotic $\left(\mathrm{AV}^{+}\right)$cells (Fig. 5a). Concurrent Cuxl haploinsufficiency and Flt3 $3^{I T D}$ mutation was associated with a further reduction in late-apoptotic $\left(\mathrm{AV}^{+} \mathrm{FVD}^{+}\right)$cells than observed in single-allele mutant groups (Fig. 5b). We also examined the frequency of apoptotic cells within the SLAM compartment of LSK cells to find reductions in early- and late-apoptotic cells in MPP cells of Cux $1^{+/-}$, Flt3 $3^{I T D}$ and Cux1 $1^{+/-} ;$Flt $3^{\text {ITD }}$ cells (Fig. 5c, d). While the frequency of apoptotic cells appeared to be lower in $\mathrm{Cux} \mathrm{I}^{+/-}$; Flt $3^{I T D}$ ST-HSCs compared with control, the paucity of these cells (and LT-HSCs) in Cux1 ${ }^{+/-} ;$Flt3 ${ }^{I T D}$ mice may have hindered their analysis (Supplementary Fig. 4a-d). Assessment of apoptosis in LK cells revealed reductions in early- and/or late-apoptotic cells in Cux1 $1^{+/-}$, Flt3 $3^{\text {ITD }}$ and Cux1 $1^{+/-} ;$Flt $3^{\text {ITD }}$ mice compared with control (Fig. 5e, f), which was mainly attributable to decreased early- and late-apoptosis in GMP cells over CMPs and MEPs 

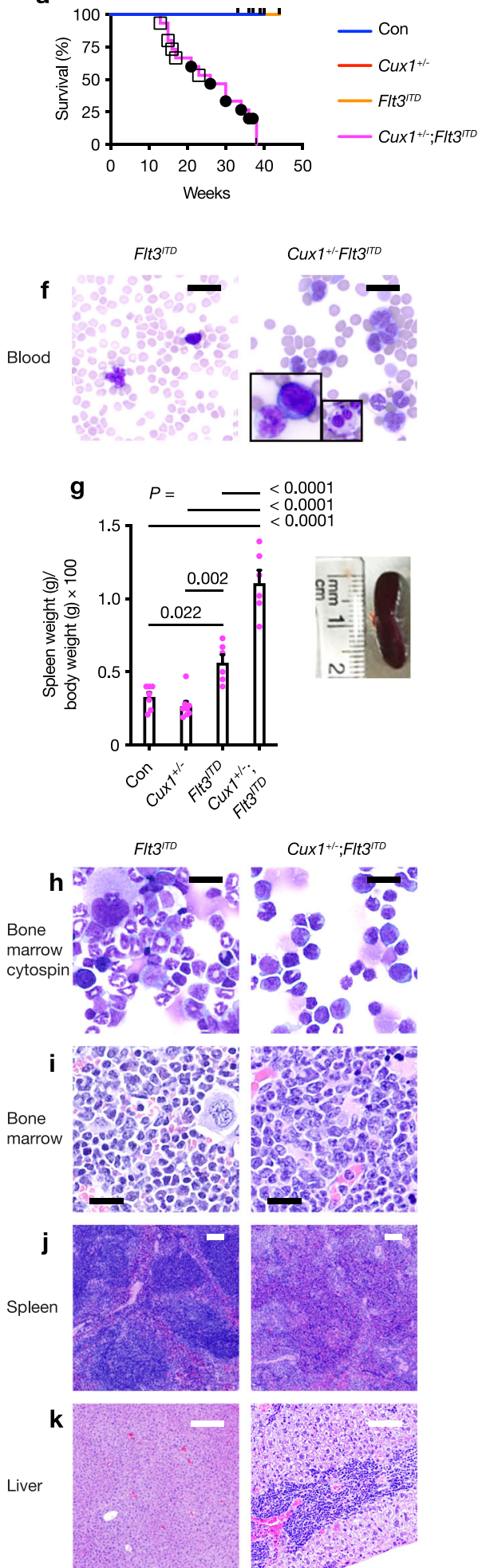
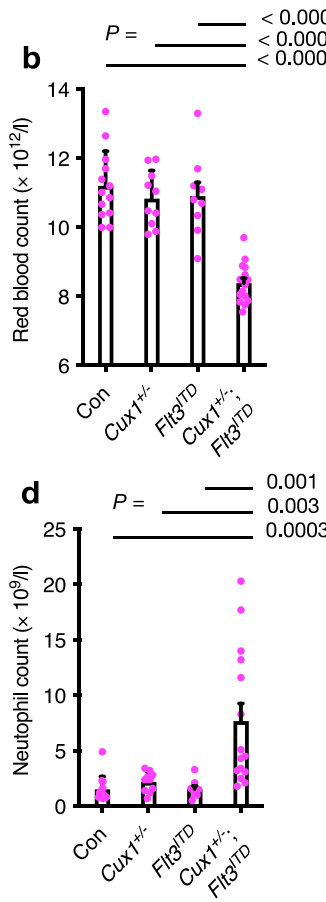

I

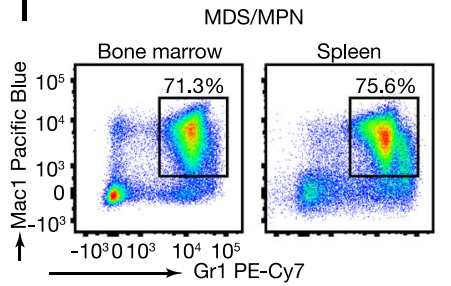

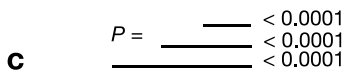
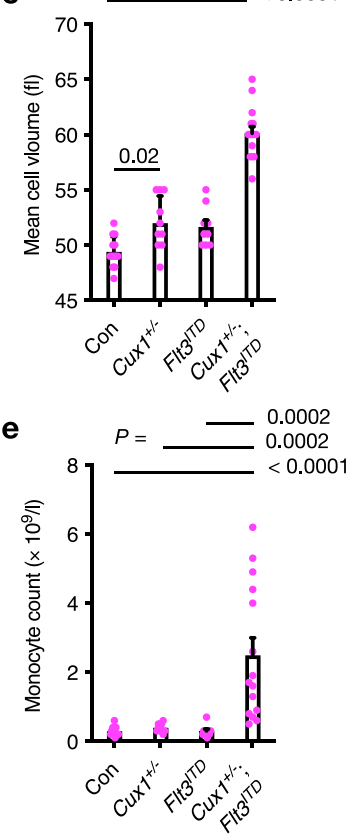

MDS/MPN

m

MPO stain

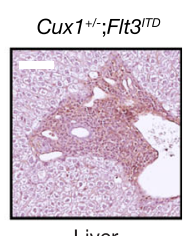

n

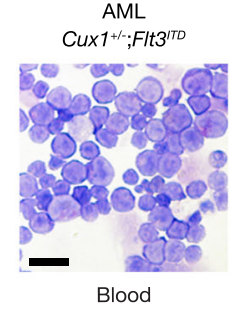

- AML

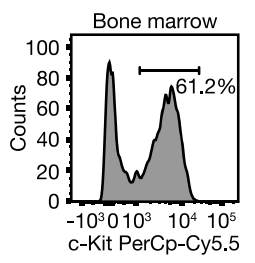

(Fig. 5g, h and Supplementary Fig. 4e-h). When the higher bone marrow cellularity of $\mathrm{Cu} \times 1^{+/-} ;$Flt $3^{I T D}$ mice was taken into account, reductions in early- and late-apoptotic absolute cell numbers were most marked in LT-HSC and ST-HSC cells of these mice (Supplementary Fig. 4i-t). To explore the status of apoptosis in hematopoietic subsets using an alternative approach, we performed flow cytometry using antibodies against the active form of caspase-3 (C3). Using this approach, we confirmed a reduction in early- $\left(\mathrm{C}^{+}\right)$and late- $\left(\mathrm{C}^{+} \mathrm{FVD}^{+}\right)$apoptotic cells in LSK cells from Cux1 ${ }^{+/-} ;$Flt3 $3^{I T D}$ mice consistent with our Annexin-V data, which we attributed to the MPP subcompartment (Supplementary Fig 4). The frequency of early-apoptotic cells was also lower in LK cells, and in particular GMPs, from Cux $1^{+/-} ; F l t 3^{I T D}$ mice, although the changes appeared to be less 
Fig. 2 F/t3 ${ }^{\text {ITD }}$ mutation cooperates with Cux1 haploinsufficiency to induce MDS/MPN and AML in vivo. a Kaplan-Meier survival curve of $C 4 x 1^{+/ /-; F l t 3 / T D}$ $\left(n=14\right.$, magenta line), Flt3 ${ }^{\text {ITD }}\left(n=9\right.$, orange line), Cux $1^{+/ f l} ; V_{\text {av-iCre }}+\left(\mathrm{Cux} 1^{+/-}\right)\left(n=11\right.$, red line) and control (Vav-iCre ${ }^{+/-}$or Cre-negative littermates,

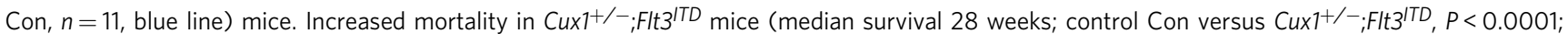

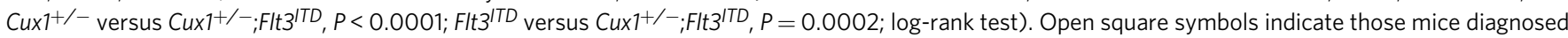
with MDS/MPN and black circles indicate AML cases. b-e Red blood cell count (b), red blood cell mean cell volume (c) neutrophil count (d) and monocyte

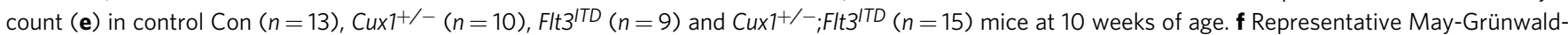
Giemsa (MGG)-stained blood smears from 10-week-old Flt3lTD (left) and Cux $1^{+/-; F I t 3 / T D}$ (right) mice. Insets show an abnormal monocyte and blast cell

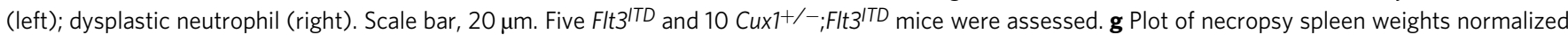

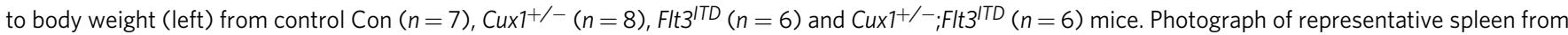

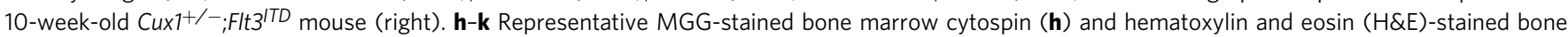
marrow (i), spleen (j) and liver ( $\mathbf{k}$ ) sections from 17-week-old Flt3 ITD (left) and Cux $1^{+/-} ;$Flt3 ${ }^{\text {ITD }}$ (right) mice. Bone marrow and tissues are infiltrated with

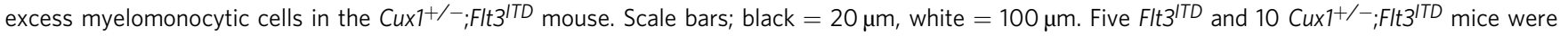
assessed. I Flow cytometric plot showing distribution of $\mathrm{Gr}^{+}$and $\mathrm{Macl}^{+}$cells in bone marrow (left) and spleen (right) from a 15-week-old Cux $1^{+/ /}$; Fltz $3^{\text {ITD }}$ mouse diagnosed with CMML. Percentage of $\mathrm{Gr}^{+} \mathrm{Macl}^{+}$cells is shown. $\mathbf{m}$ Immunohistochemistry of liver section from a 15-week-old Cux ${ }^{+/ /-}$; Flt3 $3^{\text {ITD }}$ mouse diagnosed with MDS/MPN showing infiltration with myeloperoxidase (MPO)-expressing cells. Scale bar, $100 \mu \mathrm{m}$. Three mice were assessed. n Representative MGG-stained blood smear showing blast cells from a 30-week-old Cux $7^{+/-}$;Flt3 ${ }^{\text {ITD }}$ mouse diagnosed with AML. Scale bar, $20 \mu$ m. Five mice were assessed. o Flow cytometric plot showing percentage of c-Kit ${ }^{+}$AML cells in bone marrow from a 30-week-old Cux $7^{+/-; F l t 3 / T D}$ mouse diagnosed with AML. All plots show mean + s.e.m. Each circle represents one mouse. One-way ANOVA with Tukey's test for multiple comparisons.

marked compared with those observed using Annexin-V staining (Supplementary Fig. 4). Collectively, these data demonstrate that apoptosis is perturbed in hematopoietic stem and progenitor compartments of Cux1 $1^{+/-} ;$Flt $3^{I T D}$ mice.

Using similar staining approaches, we assessed the apoptosis status within bone marrow lineage compartments. While the frequency of bone marrow $\mathrm{B} 220^{+} \mathrm{B}$ cells was reduced in Cux $1^{+/-}$;Flt3 ITD mice, probably due to the established effect of Flt ${ }^{I T D}$ expression on B-cell development ${ }^{40}$, we did not observe any significant changes in apoptosis in these cells (Supplementary Fig. 5a, c). In contrast, Annexin- $\mathrm{V}^{+}$cells were increased in

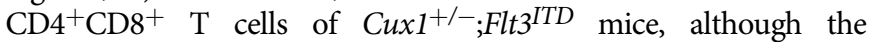
frequency of $\mathrm{T}$ cells was similar to control (Supplementary Fig. 5b, d). Intriguingly, apoptosis analysis of $\mathrm{Gr}^{+} \mathrm{Mac1}^{+}$myeloid cells was conflicting using Annexin- $\mathrm{V}$ and active caspase- 3 staining suggesting that apoptosis may not play an important role in the expansion of mature myeloid cells (Supplementary Fig. 5e, h).

To investigate erythropoiesis, we stained bone marrow cells for CD71 and Ter119 antigens to distinguish the distribution of erythroid cells along an established stage I-IV maturation pathway ${ }^{46}$. These markers are able to resolve several erythroid populations: stage I proerythroblasts (CD71 $1^{\text {high}}$;Ter119med, stage I); stage II basophilic erythroblasts (CD71 high;Ter119high); stage III polychromatophilic erythroblasts (CD71 ${ }^{\text {med;Ter119high); }}$ and stage IV orthochromatophilic erythroblasts (CD71 low; Ter119 $\left.{ }^{\text {high }}\right)$. While Cux1 haploinsufficiency led to a reduction in stage II progenitors, there was no difference in the frequency of Annexin- $\mathrm{V}^{+}$cells compared with control (Supplementary Fig. 5l). Concurrent Cuxl haploinsufficiency and Flt $3^{I T D}$ mutation led to reduced frequency of stage II-IV progenitors compared with other groups, consistent with the anemia observed in these mice and alluding a very early defect in erythropoiesis (Supplementary Fig. 5i, j). Intriguingly, the proportion of Annexin- $\mathrm{V}^{+}$cells was reduced in cells from each stage suggesting that non-apoptotic mechanisms are responsible for defective erythropoiesis and anemia (Supplementary Fig. 5k-n).

Cell cycle progression and transcriptome changes in $\mathrm{CuxI}^{+/-}$; Flt $^{I T D}$ cells. Given the reduction in LT-HSCs in Flt3 ${ }^{I T D}$ mice has been partly attributed to loss of stem cell quiescence in this cell population $^{42}$, we assessed the cell cycle status of HSCs and MPs

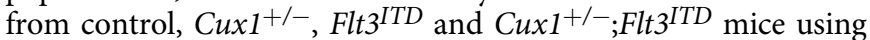
Ki-67 and DAPI staining. More LSK cells from Cux $1^{+/-} ;$Flt $3^{I T D}$ mice were in $\mathrm{G} 1$ and $\mathrm{S} / \mathrm{G} 2 / \mathrm{M}$ phases of the cell cycle compared with other groups indicating a loss of stem cell quiescence (Supplementary Fig. 6a). Using SLAM markers, we found that cell cycle progression was most pronounced in MPP cells with LTHSCs being affected to a lesser extent, while ST-HSCs did not demonstrate any abnormalities in the distribution of G0 and G1 cells (Supplementary Fig. 6b-d). Analysis of myeloid progenitors revealed a similar fall in the proportion of G0 cells in Cux $1^{+/-}$;Flt3 $3^{I T D}$ mice with a concomitant increase in G1 cells (Supplementary Fig. 6e) which affected GMPs, CMPs and MEPs (Supplementary Fig. 6f-h). Taken together, these data reveal abnormal loss of hematopoietic stem and progenitor cell quiescence in $\mathrm{Cux} 1^{+/-} ;$Flt $3^{I T D}$ mice which could contribute to both the depletion of stem cells and proliferation of more differentiated hematopoietic cell types.

To investigate further the basis of the cooperative leukemic phenotype between Flt3 ${ }^{I T D}$ and Cux1 haploinsufficiency, we performed transcriptome analysis of LSK cells isolated from Cux $1^{+/-} ;$Flt3 $3^{I T D}$ and Flt3 ${ }^{I T D}$ mice. We observed upregulation and downregulation of 297 and 347 genes respectively between Cux $1^{+/-} ;$Flt $3^{\text {ITD }}$ and Flt3 ${ }^{\text {ITD }}$ LSK cells (fold change $\geq 1.5, P_{\text {adj }} \leq$ 0.05; Supplementary Data 3). Gene ontology analysis revealed that $C u x 1^{+/-}$;Flt3 $3^{\text {ITD }}$ LSK cells exhibited an enrichment in genes involved in apoptosis, intracellular cell signaling, cell proliferation and differentiation compared with Flt ${ }^{I T D}$ cells (Supplementary Fig. 7a), while gene set enrichment analysis (GSEA) demonstrated that $C u \times 1^{+/-} ; F l t 3^{I T D}$ LSK cells displayed an enrichment in myeloid cell development and monocyte differentiation signatures (Supplementary Fig. 7b). Collectively, these analyses are in keeping with the increased myelomonocytic lineage output and phenotypes observed in $C u x 1^{+/-} ; F l t 3^{I T D}$ hematopoietic stem and myeloid progenitor cells.

CFLAR restrains apoptosis in primary $C U X 1$-haploinsufficient hematopoietic cells and represents an in vivo therapeutic target. Having established that CFLAR depletion triggers apoptotic cell death in U937 and THP-1 myeloid cancer cell lines, we sought to test the relevance of our findings in our Cuxl-haploinsufficient murine leukemia model. We determined CFLAR levels in $\mathrm{Macl}^{+}$myeloid and whole bone marrow cells from Cux $1^{+/-}$;Flt3 $3^{I T D}$ and Flt3 ${ }^{I T D}$ mice. Consistent with our observations in human myeloid cancer cell lines, Cflar transcript and corresponding protein levels were increased in $\mathrm{Cux1^{+/- }}$; Flt $3^{I T D}$ compared with Flt3 $3^{I T D}$ cells (Fig. 6a, b). We next assessed the impact of depleting Cflar using shRNA by infecting c-Kit ${ }^{+}$ 
a

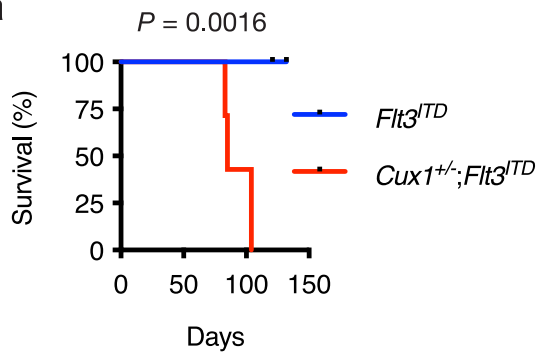

b

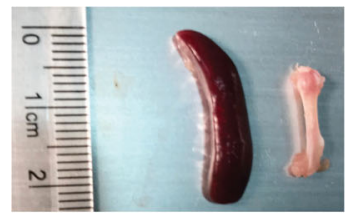

C

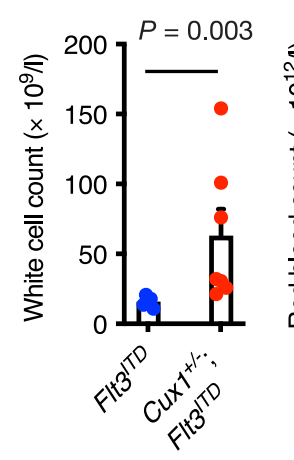

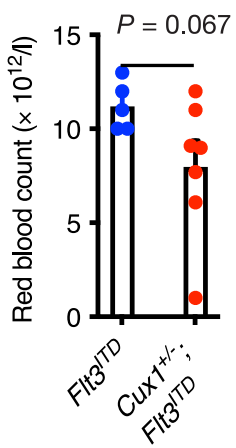

e

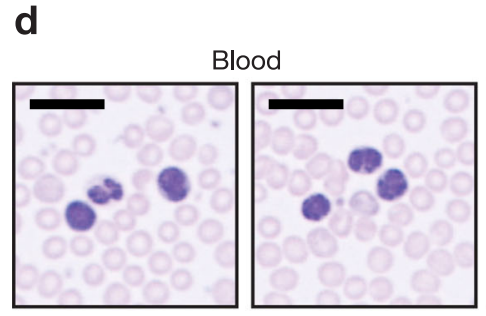

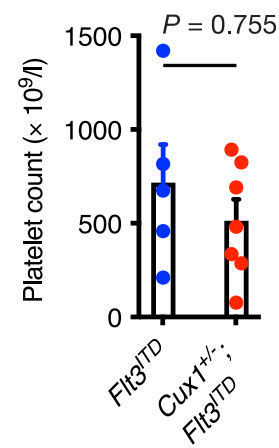

$\mathbf{f}$

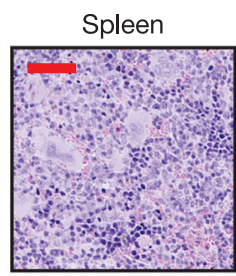

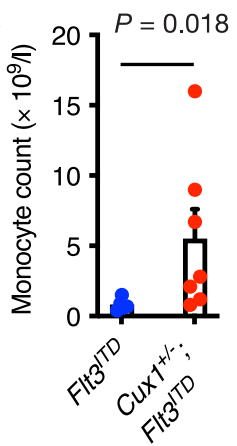

9

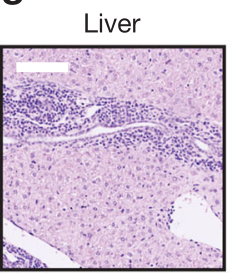

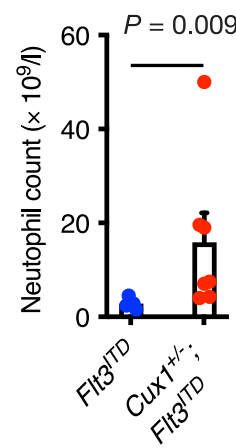

h

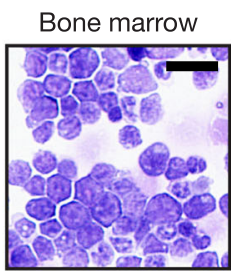

i
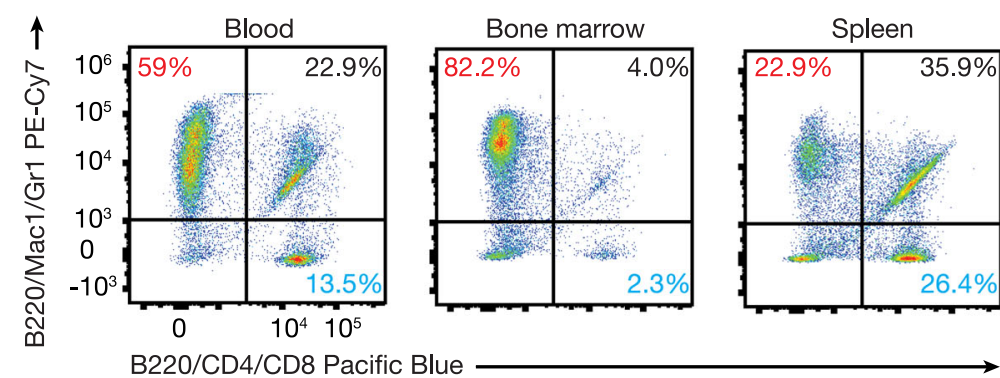

j

Flt3 $3^{1 T D}$ Cux $1^{+/-F / t 3 / T D}$
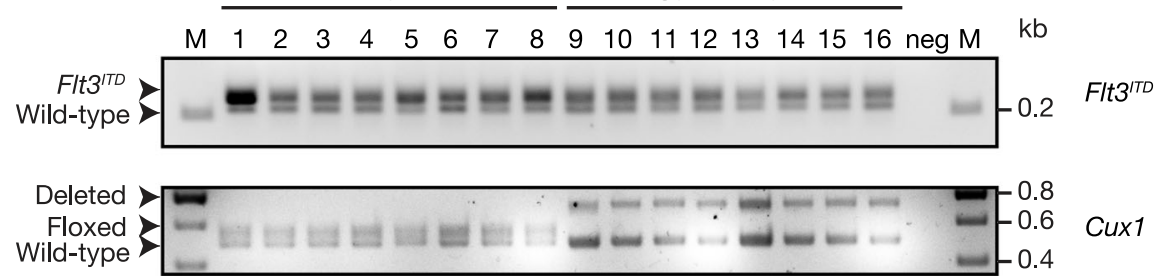

Cux1 hematopoietic cells from control (Con), Cux $1^{+/-}, \mathrm{Flt}^{I T D}$ and Cux $1^{+/-}$;Flt $3^{I T D}$ mice with lentiviruses designed to express doxycycline-inducible shRNA targeting Cflar. We cultured infected cells in the presence of doxycycline to induce Cflar knockdown or drug vehicle as a control. After $48 \mathrm{~h}$, we observed a significant reduction in live cell numbers in $\mathrm{Cux} 1^{+/-}$; Flt $3^{I T D}$ cells compared with other groups following doxycycline treatment
(Fig. 6c). A lesser effect was also seen in Cux $1^{+/-}$cells (Fig. 6c). Using Annexin-V/DAPI staining, we established that there were greater numbers of early-apoptotic $\left(\mathrm{AV}^{+}\right)$cells in doxycycline-

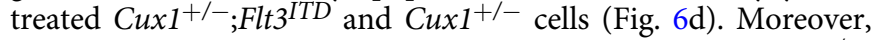
late-apoptotic cells $\left(\mathrm{AV}^{+} \mathrm{DAPI}^{+}\right)$were increased in $\mathrm{Cux1^{+/ }}{ }^{+}$; Flt $3^{I T D}$ cells compared with control (Fig. 6d). The difference in cell numbers was also apparent in methylcellulose assays, where 


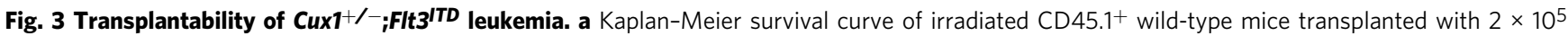
Cux $7^{+/-} ;$Flt3 ITD $(n=7)$ or Flt3 ITD $(n=5)$ bone marrow cells, along with the same number of CD45.1+ wild-type support bone marrow cells. Bone marrow cells from two Cux ${ }^{+/-} ;$FIt3 $3^{I T D}$ mice diagnosed with $\mathrm{AML}$ at 30 weeks of age were injected into three or four irradiated recipients. Flt3/TD donor cells were

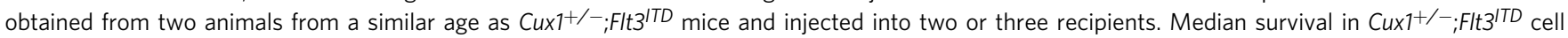
recipients was 85 days. $P=0.0016$, log-rank test. b Representative photograph of an enlarged spleen (left) and pale femur (right) from AML-diseased 85 day-old mouse transplanted with Cux $1^{+/-; F I t 3 / T D}$ bone marrow cells. c Blood count parameters from mice transplanted with cells of the indicated genotypes at 12 weeks post transplantation. Higher white cell counts due to increased monocyte and neutrophil numbers are seen in mice transplanted with $\mathrm{Cux} 1^{+/-}$; FIt3 $3^{\text {TD }}$ bone marrow cells. d-g MGG-stained blood smears (d) showing abnormal circulating neutrophils and monocytes and H\&E-stained bone marrow (e), spleen (f) and liver ( $\mathbf{g}$ ) sections showing myelomonocytic cell tissue infiltration compromising normal tissue architecture. Five mice were assessed. h MGG-stained bone marrow cytospin showing AML blasts from an 85 -day-old mouse transplanted with Cux $1^{+/-;}$;It3 $3^{\text {ITD }}$ bone marrow cells. Scale bars; black $=20 \mu \mathrm{m}$, red $=50 \mu \mathrm{m}$, white $=100 \mu \mathrm{m}$. Five mice were assessed. $\mathbf{i}$ Flow cytometric plots showing increased proportion of myeloid $\left(\mathrm{Gr}^{+} \mathrm{Mac1}^{+}\right.$, red) cells within blood, bone marrow and spleen. The proportions of B220+ $\mathrm{B}$ cells (black) and $\mathrm{CD} 4^{+} \mathrm{CD} 8^{+} \mathrm{T}$ cells (blue) are also shown.

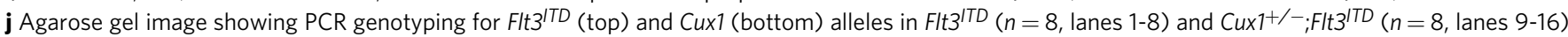
mice. M, DNA ladder; neg, no DNA template. Identity of PCR products is shown on the left. Each circle represents one mouse. Plots show mean + s.e.m. Two-tailed, unpaired Mann-Whitney test.

we observed fewer colonies arising from doxycycline-treated

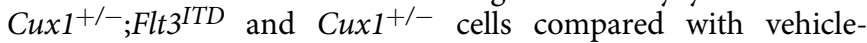
treated cells after seven days (Fig. 6e). Importantly, there was no significant difference in colony number from control or Flt $3^{I T D}$ cells treated in the same way, arguing that Cflar is a specific vulnerability in the context of Cux1 haploinsufficiency (Fig. 6e). To investigate the contribution of apoptosis further, we examined the status of the initiator and executioner caspases downstream of the canonical CFLAR apoptosis pathway following Cflar deple-

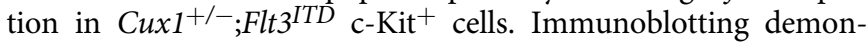
strated that Cflar depletion was associated with activation and cleavage of caspase- 3 and caspase- 8 proteins, consistent with the established role of CFLAR in inhibiting the initiator caspase, caspase-8 (Fig. 6f). In contrast, retroviral restoration of CFLAR in Flt ${ }^{I T D} \mathrm{c}^{\mathrm{C}} \mathrm{Kit}^{+}$cells had the opposite effect on caspase activation (Fig. 6g).

Since CFLAR can also influence cell death through necroptosis, we assessed the contribution of necroptosis to cell death in our model. To this end, we treated $\mathrm{c}-\mathrm{Kit}^{+}$cells from each genotype group with a combination of doxycycline and the RIPK1 inhibitor, Necrostatin-1 (Nec-1). We found that Nec-1 treatment was unable to rescue cell viability in doxycycline-treated cells, arguing that necroptosis does not play a significant role in cell death following Cflar depletion (Fig. 6c). Furthermore, we did not observe any evidence of MLKL phosphorylation in doxycyclinetreated ${\mathrm{c}-\mathrm{Kit}^{+}}^{+}$cells from any genotype, again suggesting apoptosis rather than necroptosis is the predominant means of cell death following Cflar depletion in our model (Supplementary Fig. 8).

To assess the impact of Cflar reduction on leukemia development in vivo, we performed transplant experiments

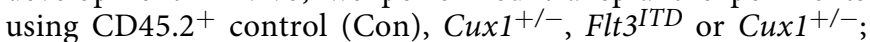
Flt3 $^{I T D} \mathrm{c}^{-\mathrm{Kit}^{+}}$cells transduced with doxycycline-inducible, Cflar-targeting shRNA lentiviruses along with wild-type CD $45.1^{+}$CD $45.2^{+}$support bone marrow cells into lethally irradiated $\mathrm{CD} 45.1^{+} \mathrm{CD} 45.2^{+}$recipient mice (Fig. 6h). Four weeks following transplant, we administered doxycycline chow for two weeks to experimental groups, while control groups were maintained on a normal diet. Flow cytometric analysis revealed that the frequency of transplanted, bone marrow CD $45.2^{+}$cells was significantly reduced in mice injected with Cux $1^{+/-}$or Cux1 $1^{+/-} ; F_{1 t 3} 3^{I T D}$ cells only following doxycycline chow administration compared with mice injected with control or Flt3 ${ }^{I T D}$ cells (Fig. 6i). Furthermore, spleen weights were significantly reduced in doxycycline-treated mice injected with $\mathrm{Cux} 1^{+/-}$or $\mathrm{Cux1^{+/- }}$ Flt3 $3^{\text {ITD }}$ cells transduced with Cflar-targeting shRNAs compared with control and Flt3 $3^{I T D}$ groups (Fig. 6j). Collectively, our data support the hypothesis that Cflar acts to safeguard against apoptosis in Cux1haploinsufficient cells.

CUX1 is a direct transcriptional repressor of CFLAR expression. Since our data supported the idea that CFLAR is a mediator of protective, anti-apoptotic responses in CUX1-deficient cells, we sought to determine the basis of this phenomenon and noticed that CUX1-1- U937 cells displayed higher levels of CFLAR protein expression and cytarabine resistance (Fig. 7a and Supplementary Fig. 9a). CFLAR and CUX1 levels were also reciprocally correlated in tested human AML cell lines and in CUX1knockdown Cancer Cell Line Encyclopedia efforts ${ }^{47}$ (Supplementary Fig. 9b, c). Reverse-phase protein array (RPPA) analysis suggested that CUX1-l- cells exhibited higher pAKT ${ }^{S 473}$ and pAKT ${ }^{\mathrm{T} 308}$ levels consistent with previously reported AKT pathway activation ${ }^{18}$ (Supplementary Fig. 9d and Supplementary Data 4). In contrast, cleaved caspases-8 levels appeared reduced in agreement with the anti-apoptotic function of CFLAR (Supplementary Fig. 9d, e). Higher transcript levels of CFLAR and CYBB (whose expression is known to be repressed by CUX1; ref. ${ }^{48}$ ) were also observed (Fig. 7b), leading us to hypothesize that CUX1 acts as a direct transcriptional repressor of CFLAR. Induction of CUX1p110 or CUX1 $1^{200}$ protein isoforms, which can be biotinylated, to near physiologic levels or Cherry protein control in CUX1-/- U937 cells led to suppression of CFLAR by CUX1 $1^{\text {200 }}$ but not CUX1 $1^{110}$ or Cherry, consistent with CUX1p200 acting as a repressor of CFLAR expression (Fig. 7c and Supplementary Fig. 9f). Furthermore, promoter-luciferase assays using a $\sim 4 \mathrm{~kb}$ genomic fragment upstream of the CFLAR transcriptional start site (TSS), which harbors multiple putative CUX1-binding sites similar to the consensus ATYGATSSS (where $\mathrm{Y}=\mathrm{C} / \mathrm{T}, \mathrm{S}=\mathrm{C} / \mathrm{G}$ ), showed that CUX1 ${ }^{200}$ was able to repress promoter-luciferase activity (Fig. 7d). Moreover, assays using deletion constructs narrowed the repressive activity of $\mathrm{CUX}_{1} \mathrm{p}^{200}$ to a $275 \mathrm{bp}$ region upstream of the TSS, which contains two putative CUX1-binding sites (Fig. 7e, f). By using electrophoretic mobility shift assays, we found that CUX1 $1{ }^{200}$ was only able to bind the putative CUX1binding site nearest to the TSS in specific manner as revealed by cold probe and antibody competition assays (Fig. $7 \mathrm{~g}$ ). Mutation of this site within the promoter construct abolished CUX1 ${ }^{200}$ mediated repression of promoter-luciferase activity (Fig. 7e). Chromatin immunoprecipitation assays followed by qRT-PCR for this and distal control genomic regions using doxycyclineinducible CUX1 ${ }^{\mathrm{p} 110}, \mathrm{CUX}_{1} \mathrm{p}^{200}$ and control cell lines showed that CUX1 $1^{200}$ binds selectively to -132 to -122 region of the CFLAR promoter in vivo (Fig. $7 \mathrm{~h}$ ). In summary, these data establish CUX1 $1^{200}$ as a direct repressor of CFLAR expression in vivo and 

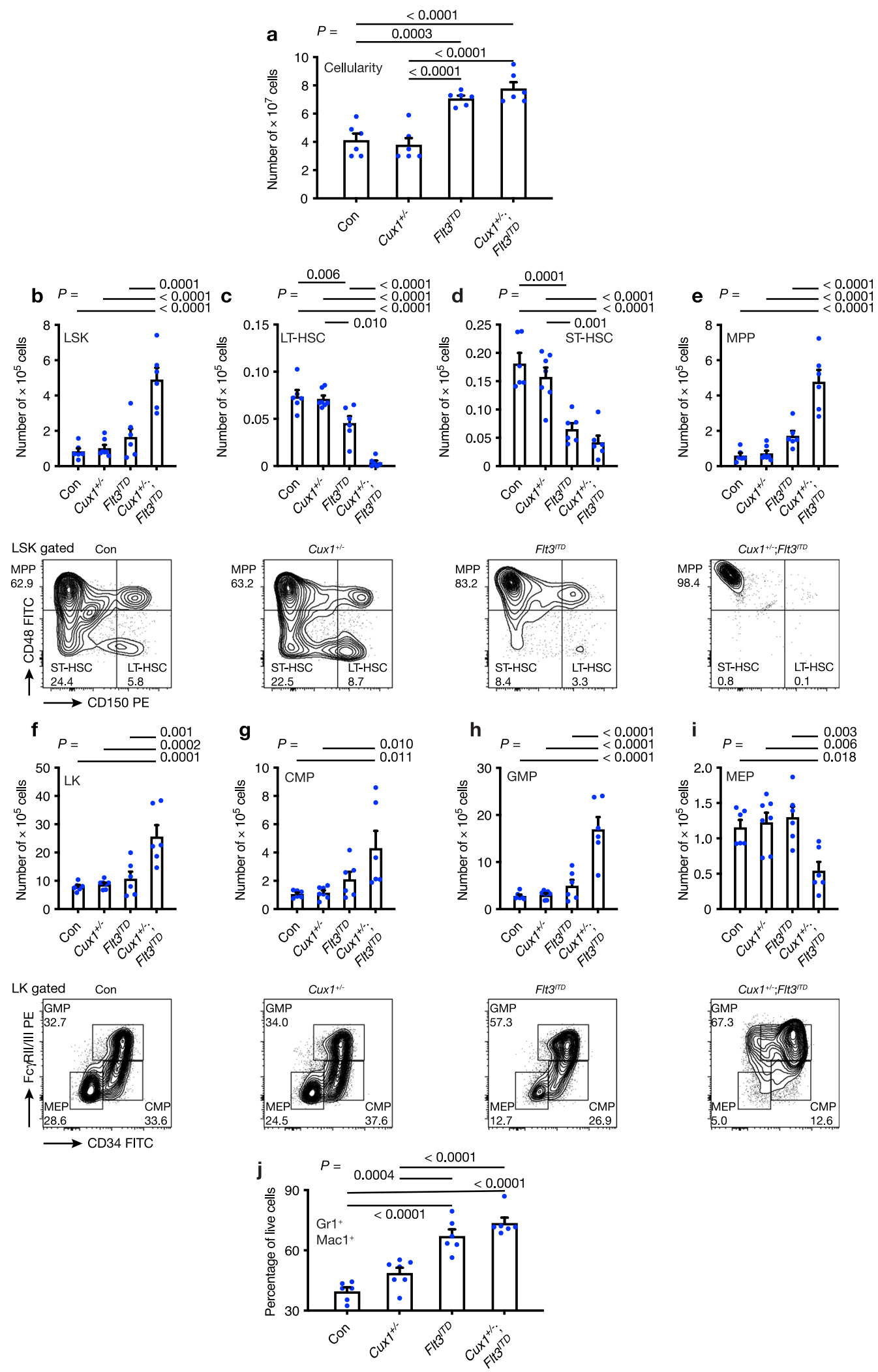

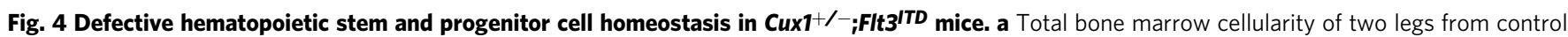

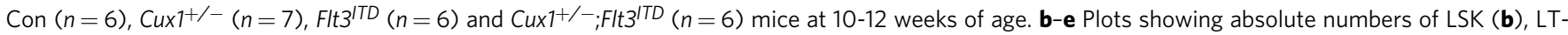
HSC (c), ST-HSC (d) and MPP (e) cells in mice from the indicated genotypes. Representative flow cytometry plots are shown below each bar plot. (f-i) Plots showing absolute numbers of LK (f), CMP (g), GMP (h) and MEP (i) cells in mice from the indicated genotypes. Representative flow cytometry plots are shown below each bar plot. $\mathbf{j}$ Percentage of $\mathrm{Gr}^{+}{ }^{+}$Mac1 $1^{+}$myeloid cells in the bone marrow of mice from the indicated genotypes ( $n=6$ per group). All plots show mean + s.e.m. Each circle represents one mouse. One-way ANOVA with Tukey's test for multiple comparisons. 
a

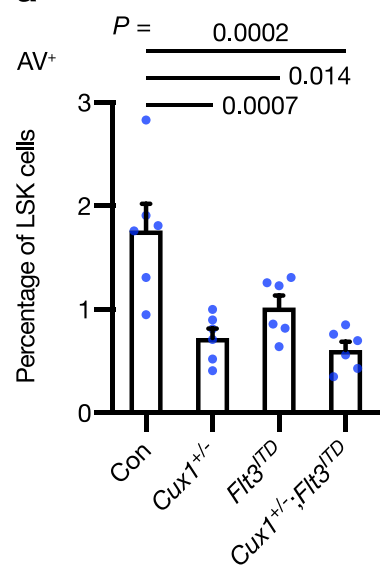

e

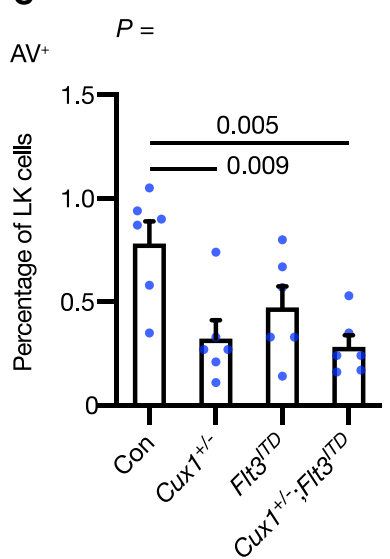

b

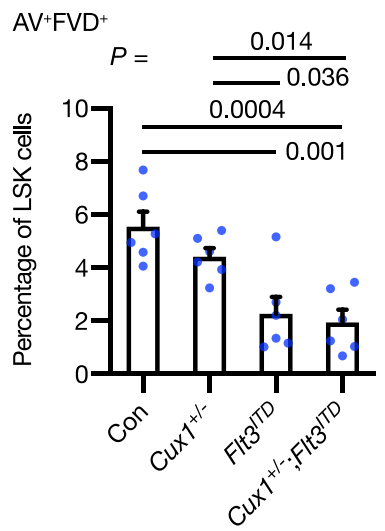

f

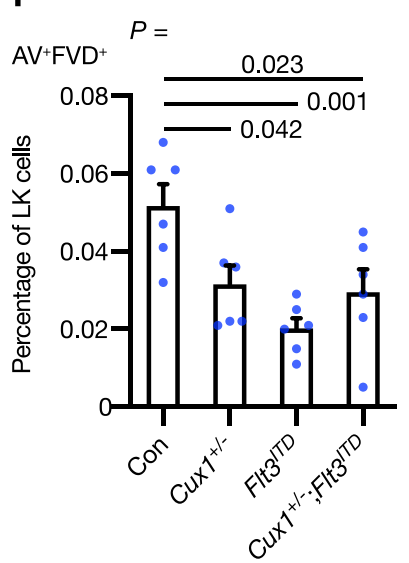

C

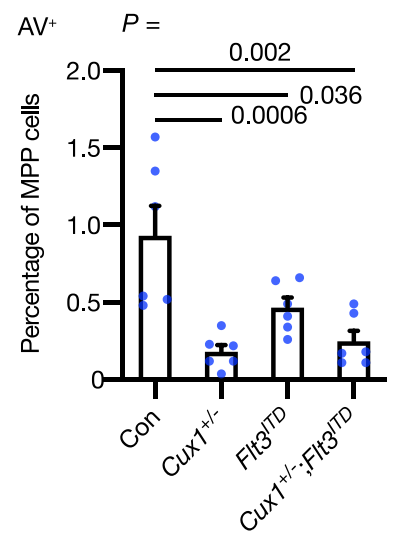

g

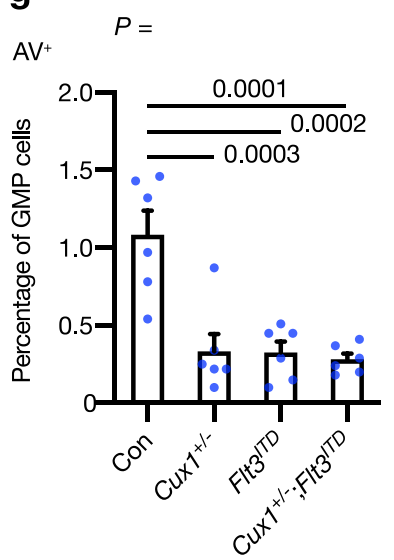

d

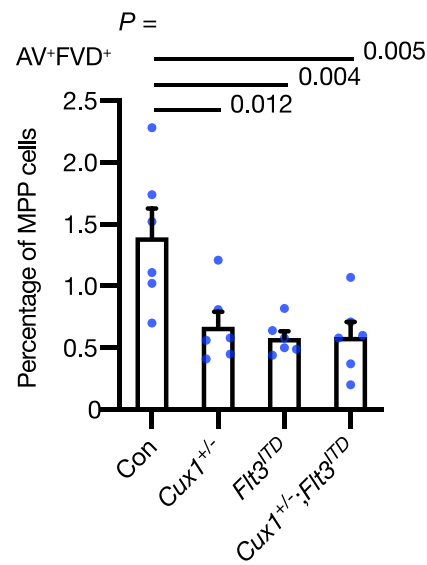

h

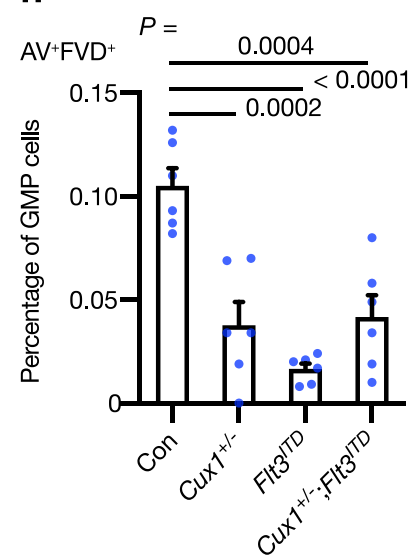

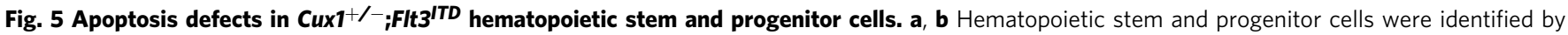
flow cytometry and apoptosis within each compartment was determined by staining with Annexin-V (AV) and a fixable viability dye (FVD). Percentage of

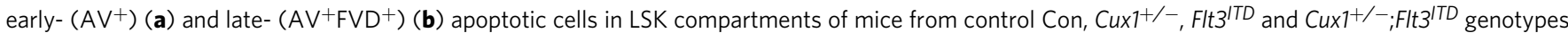
at 10-12 weeks of age ( $n=6$ per group). c-h Percentage of $\mathrm{AV}^{+}(\mathbf{c}, \mathbf{e}, \mathbf{g})$ and $\mathrm{AV}^{+} \mathrm{FVD}+(\mathbf{d}, \mathbf{f}, \mathbf{h})$ apoptotic cells in MPP (c, d), LK (e, f) and GMP ( $\left.\mathbf{g}, \mathbf{h}\right)$ compartments of mice from the indicated genotypes at 10-12 weeks of age ( $n=6$ per group). All plots show mean + s.e.m. Each circle represents one mouse. One-way ANOVA with Tukey's test for multiple comparisons.

imply that CUX1 deficiency can lead to CFLAR upregulation and associated apoptosis attenuation.

Primary -7/del(7q) AML cells are sensitive to CFLAR-signaling axis inhibition. To determine whether CUX1 deficiency led to similar CFLAR dependency in human hematopoietic cells, we designed lentiviral vectors to co-express CUX1-targeting shRNAs and GFP or CFLAR-targeting shRNAs along with mCherry. Vectors expressing non-targeting shRNAs linked to GFP or mCherry were generated as controls. We assessed the knockdown efficiency of a pair of shRNAs targeting CUX1 or CFLAR in U937 cells and selected shCUX1.2 that resulted in downregulated protein levels close to $50 \%$ compared with controls for further study (Fig. 8a, b). Human CD34 ${ }^{+}$cells from healthy donors were transduced with pairs of GFP- and mCherry-expressing shRNA vectors to target $C U X 1, C F L A R$ or non-targeting controls in order to achieve depletion of CUX1 and/or CFLAR. $\mathrm{GFP}^{+} \mathrm{mCherry}^{+}$ double-positive cells were isolated and their colony-forming potential was assessed in methylcellulose assays. While CUX1 or CFLAR depletion alone had no significant impact on colony numbers, combined CUX1 and CFLAR knockdown resulted in significantly fewer colonies compared with non-targeting control, consistent with our findings in our other models (Fig. 8c).
At present no pharmacological inhibitors of CFLAR are routinely available, but second mitochondria-derived activator of caspase (SMAC)-mimetics, such as birinapant, have attracted attention as therapies in cancer, including myeloid malignancies. SMAC-mimetics antagonize IAP family members including cellular IAP (cIAP) and X-linked IAP (XIAP) proteins, which act proximally and distally of CFLAR to promote caspase activation and apoptosis ${ }^{49}$, suggesting an alternative pharmacological, therapeutic avenue. Notably, XIAP was ranked at position 147 in our CRISPR/Cas9 screen ( $Z$ score $=3.13$ ), suggestive of preferentialvulnerability of CUX1-deficient cells to XIAP loss. Treatment of ${\mathrm{c}-\mathrm{Kit}^{+}}^{+}$cells from control, Cux1 $1^{+/-}$, Flt3 ${ }^{I T D}$ and $C u \times 1^{+/-} ; F l t 3^{I T D}$ mice led to dose-dependent reduction in cIAP1/2 and XIAP levels as anticipated (Fig. 8d). We cultured $\mathrm{c}-\mathrm{Kit}^{+}$cells from each genotype in the presence of various birinapant concentrations and determined the numbers of live cells after $48 \mathrm{~h}$ of treatment. There was a significant birinapant dose-dependent reduction in the number of live cells in $\mathrm{Cu} \times 1^{+/-}$and $\mathrm{Cu} \times 1^{+/-} ; \mathrm{Flt3}^{I T D}$ groups compared with the control (Fig. 8e). Furthermore, apoptosis assays revealed an increase in early- and late- apoptotic cells in these two groups following birinapant treatment (Fig. 8f). Consistent with this observation, cleaved caspase- 3 levels were increased in these cells by immunoblotting (Fig. 8d). 
a

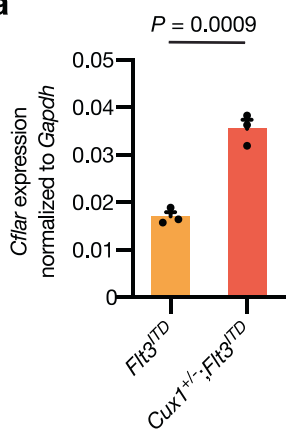

b

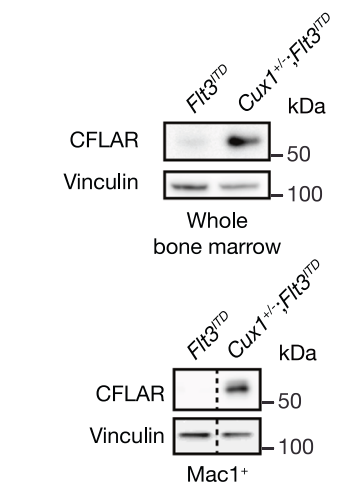

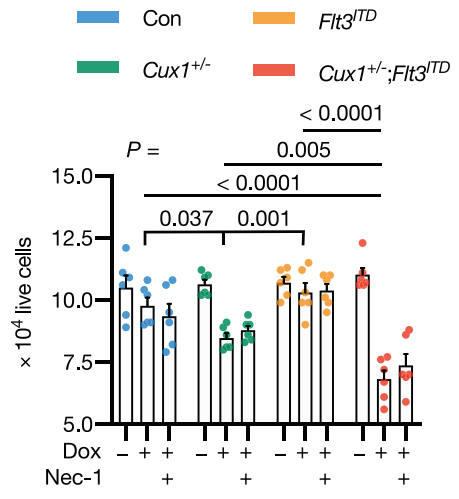

d
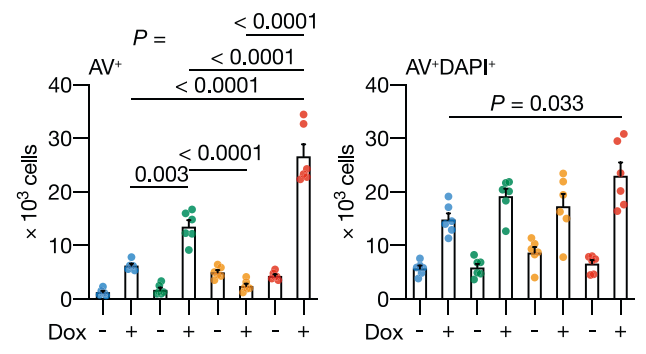

e

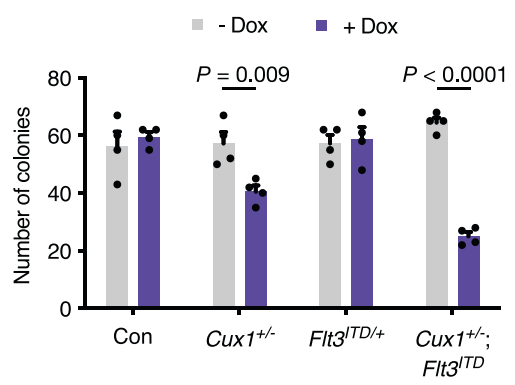

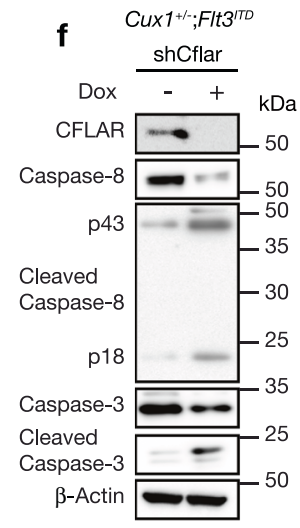

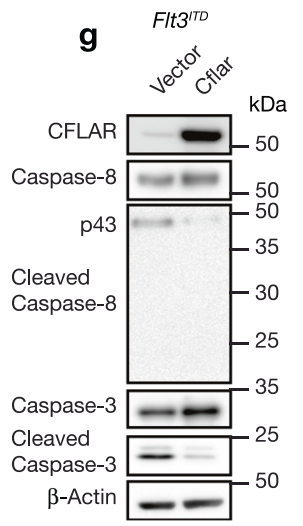

i

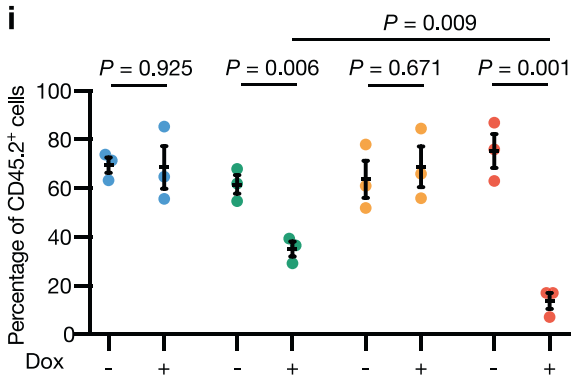

h

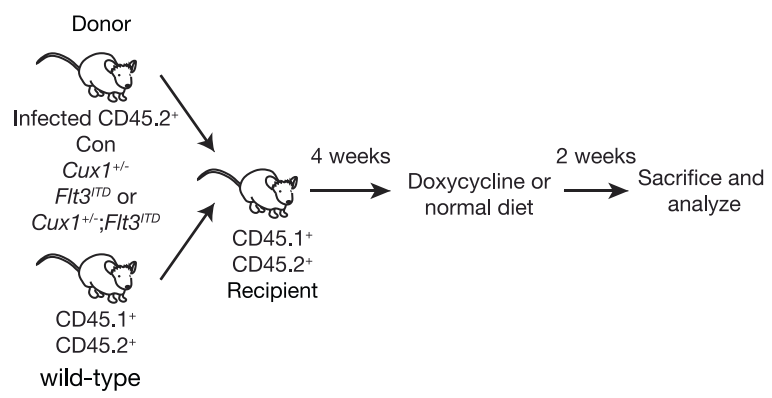

Given cIAP proteins and their antagonism can potentially affect cell survival by other mechanisms such as necroptosis and NFKB signaling ${ }^{49}$, we assessed the contribution of these pathways to birinapant-induced cell death. The addition of Nec-1 to birinapant-treated cells was unable to ameliorate the loss of viability and birinapant treatment was not associated with MLKL phosphorylation in any genotype group (Supplementary Fig. 10a).
Immunoblotting of birinapant-treated cell lysates revealed reduction in NFkB p65 and increased IкBa levels, consistent with suppression of canonical NFkB signaling. Conversely, noncanonical NFKB signaling appeared to be activated as suggested by increased NFkB p52 levels. However, there were no major differences between genotypes (Supplementary Fig. 10b). Taken together, our data show that Cux1-haploinsufficient cells exhibit 
Fig. 6 CFLAR is a genetic vulnerability and restrains apoptosis in CUX1-deficient AML. a Cflar transcript expression by qRT-PCR in Cux ${ }^{+/ /-} ;$Flt3 ${ }^{I T D}$ and Flt3 ${ }^{\text {ITD }} \mathrm{Mac1}^{+}$cells. Expression was normalized to Gapdh. Biological and technical triplicates were analyzed. b Immunoblot showing increased CFLAR

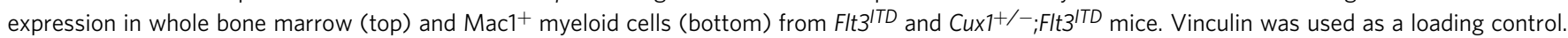
The dashed line indicates splicing of non-adjacent lanes from the same blot. The experiment was performed twice. c Growth assays using $2 \times 10^{4} \mathrm{c}-\mathrm{Kit}^{+}$ cells from two mice of the indicated genotypes plated in triplicate. Doxycycline $(0.1 \mu \mathrm{g} / \mathrm{ml}$, Dox $)$ and/or Nec- 1 ( $20 \mu \mathrm{M})$ was added and the number of live cells per well was determined by Annexin-V/DAPI staining $48 \mathrm{~h}$ later. $\mathbf{d}$ Annexin-V/DAPI staining to determine numbers of early- and late-apoptotic cells in doxycycline-treated cells from c. e Quantification of colonies derived from plating 500 c-Kit ${ }^{+}$cells transduced with doxycycline-inducible Cflar-targeting shRNA lentiviruses from indicated mice. Cells from two mice were plated in duplicate on cytokine-supplemented methylcellulose in the presence or

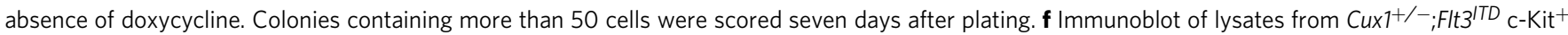
cells transduced with doxycycline-inducible Cflar-targeting shRNA lentiviruses with and without $48 \mathrm{~h}$ of doxycycline treatment showing differences in caspase- 8 and -3 activation. The immunoblot was performed once. $\mathbf{g}$ Immunoblot of lysates from Fltz ${ }^{\text {TDD }}$ c-Kit $^{+}$cells showing effect of stable CFLAR expression on caspase- 8 and -3 activation. The immunoblot was performed once. $\mathbf{h}$ Experimental outline of bone marrow transplant assay to assess

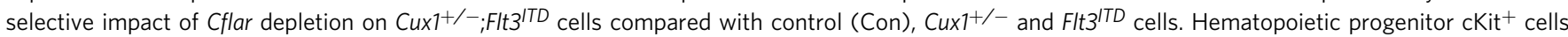
from $\mathrm{CD} 45.2^{+}$mice of each genotype were infected with doxycycline-inducible Cflar-targeting shRNA lentiviruses and $10^{5}$ puromycin-selected cells were transplanted with $10^{5}$ wild-type CD45.1+CD45.2+ bone marrow support cells into lethally irradiated CD45.1+CD45.2+ recipient mice. After four weeks, mice were fed a doxycycline-containing diet to induce Cflar depletion or maintained on a normal diet. Donor-derived CD45.2+ bone marrow cells were quantified two weeks later by flow cytometry. i Quantification of donor-derived bone marrow CD45.2+ cells in recipient mice ( $n=6$, per group) transplanted with control (Con), Cux $1^{+/-}$, Flt $3^{\text {ITD }}$ or Cux $7^{+/-} ;$Flt $3^{\text {ITD }}$ c-Kit ${ }^{+}$cells with and without doxycycline-induced Cflar depletion. $\mathbf{j}$ Spleen weights normalized to body weight of mice at the termination of the transplant. All plots show mean $+/ \pm$ s.e.m.; ns, not significant; two-way (c) or one-way (d, $\mathbf{j}$ ) ANOVA with Tukey's test for multiple comparisons, Two-tailed, unpaired $t$-test (a, e, i).

increased sensitivity to birinapant treatment, which is associated with augmented apoptosis.

Finally, to explore the relevance of our findings in primary human leukemia samples, we performed colony-forming assays using mononuclear cells isolated from AML patients with and without -7/del(7q) chromosomal lesions (Supplementary Data 5) treated with birinapant or drug vehicle. Although samples showed genetic heterogeneity and variable colony-forming capacity, the two available -7/del(7q) AML cases displayed sensitivity to birinapant treatment, while normal karyotype and healthy-donor $\mathrm{CD} 34^{+}$samples remained largely unaffected, supporting a potential relationship between CUX1 gene dosage and sensitivity to birinapant (Fig. 8g and Supplementary Fig. 10c).

\section{Discussion}

It is increasingly acknowledged that oncogenes can induce nononcogene addictions of potential therapeutic importance. Our study indicates that tumor suppressor haploinsufficiency is sufficient to induce an apoptosis stress-mitigating pathway which is essential for leukemic cell survival-a state we refer to as haploinsufficiency-induced gene addiction. Moreover, our data directly link a transcription factor (CUX1) in mediating this apoptosis-control pathway addiction through transcriptional regulation of CFLAR expression. Our study reinforces the importance of apoptosis control in myeloid leukemogenesis and particularly highlights the significance of the extrinsic apoptosis pathway in AML survival associated with CUX1 haploinsufficiency. Notably, higher CFLAR expression has been associated with inferior survival in one AML cohort ${ }^{50}$ and chemotherapy resistance in several tumor types ${ }^{51-53}$. In contrast to pancreatic cancer, where CUX1 is oncogenic, highly expressed and suppresses TRAIL-induced apoptosis through unclear mechanisms ${ }^{54}$, we propose that CUX1 haploinsufficiency in myeloid malignancies confers a reliance on CFLAR-regulated anti-apoptotic survival. How CUX1 behaves as an oncogene in some tumor types while acting as a tumor suppressor in myeloid malignancies remains unclear, but we speculate that differences in CUX1 target gene expression programs between cell types may ultimately either promote or suppress cellular transformation in a tissuespecific fashion. Uncovering crucial CUX1 target genes involved in tumorigenesis in different cell types is an important goal for future studies.
Haploinsufficiency for CUX1 due to somatic mutations is associated with inferior prognosis in myeloid malignancies similar to the adverse outcome of $-7 / \operatorname{del}(7 \mathrm{q})$ myeloid malignancies which has been recognized for over three decades ${ }^{55,56}$ Our conditional knockout mice establish that Cuxl haploinsufficiency requires cooperative mutations such as Flt ${ }^{I T D}$ to induce the spectrum of $-7 / \mathrm{del}(7 \mathrm{q})$ myeloid malignancies as observed in humans. As AML emerges from an MDS/MPN disease in our model, it will be interesting to determine whether additional genetic lesions are acquired during leukemia development. In the context of Flt3 ITD mutation, Cux1 haploinsufficiency leads to dysregulation of hematopoietic stem and progenitor cell homeostasis, which is associated with defects in apoptosis and cell cycle quiescence. Although the direct contribution of Cflar to these phenotypes requires further investigation, our data show that CFLAR safeguards against apoptotic cell death in CUX1-haploinsufficient cells.

While direct inhibitors of CFLAR are in preclinical development ${ }^{57}$, the sensitivity of CUX1-haploinsufficient cells to birinapant alludes to a potentially actionable way to treat CUX1deficient myeloid malignancies. While birinapant treatment induces apoptosis in CUX1-haploinsufficient cells, we acknowledge that other birinapant-mediated effects on processes such as NFKB signaling could also influence survival outcome. Long-term treatment of our Cux1-haploinsufficient leukemia model with birinapant will be interesting to determine the durability of responses. A previous study showed that birinapant increased apoptosis in human AML stem cells compared with $\mathrm{CD} 34^{+}$cells from normal individual ${ }^{58}$. Although a phase 2 study of azacitidine with or without birinapant failed to demonstrate a difference in response rate between the two arms in genetically unselected myeloid malignancy patient ${ }^{59}$, we encourage the development of direct CFLAR inhibitors and the further clinical evaluation of SMAC-mimetics in -7/del(7q) myeloid malignancies. Future studies will aim to examine the efficacy of combining PI3K-AKT pathway inhibition with CFLAR pathwaytargeting agents in poor-prognosis -7/del(7q) myeloid malignancies. Finally, since CUX1 is mutated in a wider spectrum of cancers ${ }^{18}$, our findings merit further scrutiny in other CUX1-mutated cancer types.

\section{Methods}

Mouse strains. Conditional Cuxl knockout mice, harboring loxP sites flanking Cuxl exons 15-18, were generated by injecting C57BL/6 N zygotes with two in 


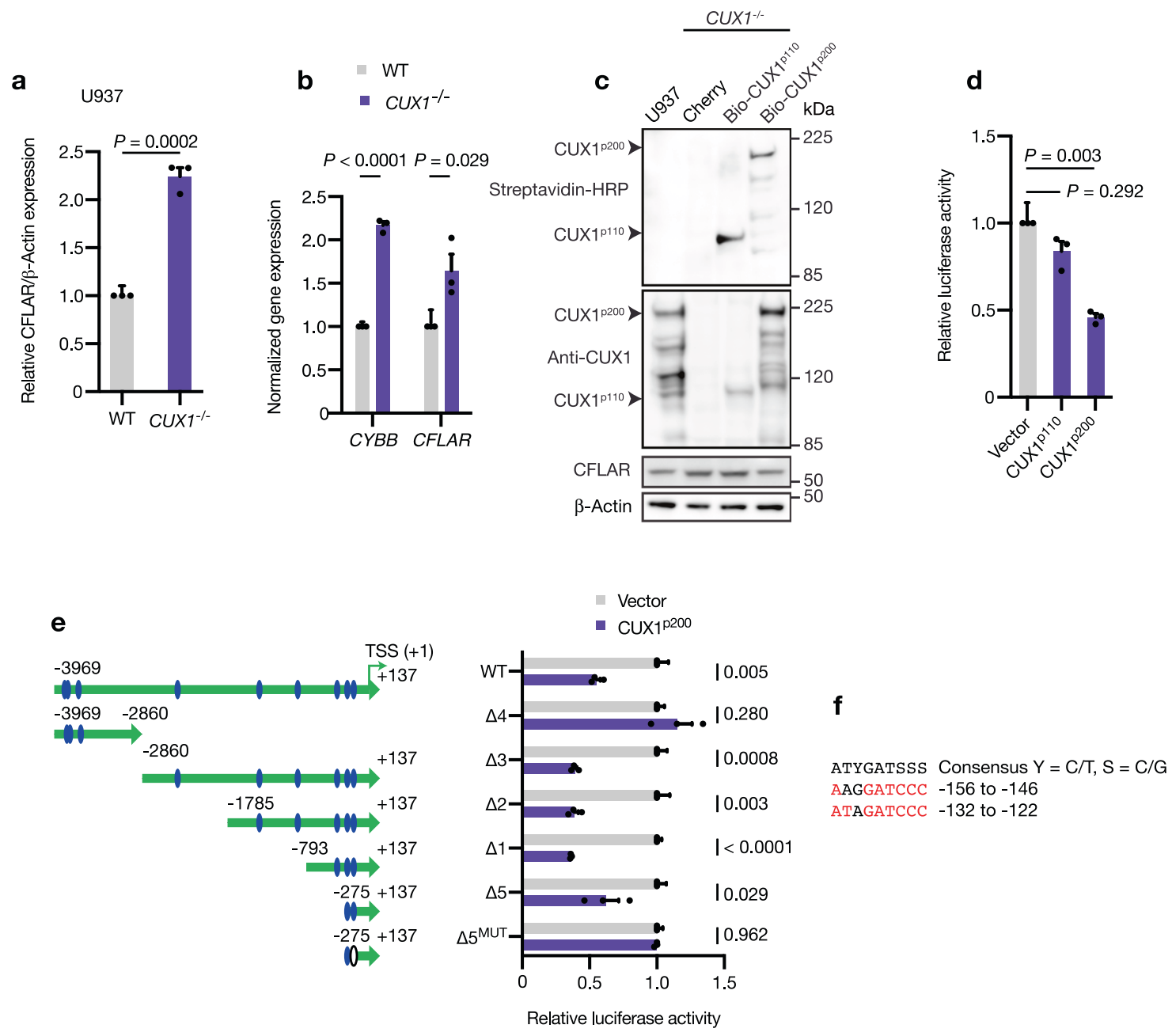

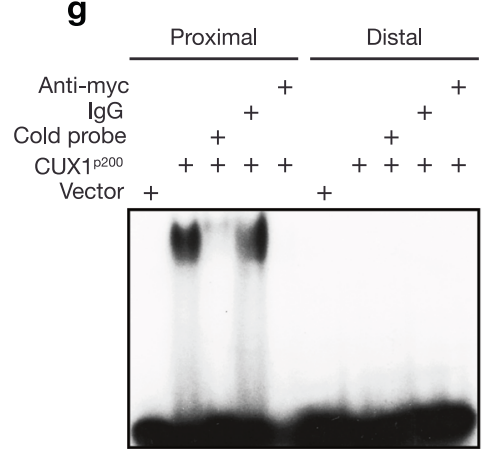

vitro-transcribed T7 promoter-primed sgRNAs $(12.5 \mathrm{ng} / \mu \mathrm{l})$ targeting introns 14 (Cux1_int14) and 18 (Cux1_int18), two complementary loxP site-containing donor single-stranded oligonucleotides (Cux1_loxP14 and Cux1_loxP18; $100 \mathrm{ng} / \mu \mathrm{l}$ ), along with in vitro-transcribed T7 promoter-primed Cas9 mRNA ( $50 \mathrm{ng} / \mu \mathrm{l})$. Oligonucleotides used in this study are listed in Supplementary Data 6. Zygotes were cultured until the blastocyst stage before transplantation into pseudo-pregnant females. Correctly targeted founder mice were identified by PCR using primers P1 and $\mathrm{P} 2$ for the first $\operatorname{lox} \mathrm{P}$ site and $\mathrm{P} 3$ and $\mathrm{P} 4$ for the second lox $\mathrm{P}$ site along with ear punch genomic DNA isolated from offspring of chimeric mice bred to C57BL/6 N wild-type animals. The identity of PCR products was confirmed by sequencing. Mice were maintained on a C57BL/6 background.

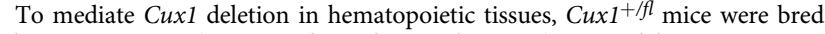
with Vav-iCre mice (008610, The Jackson Laboratory). Cux1 deletion was confirmed by PCR using primers P1, P2 and P3 along with genomic DNA from splenocytes or peripheral blood leukocytes. Wild-type, floxed and deleted Cuxl alleles led to PCR products of 502, 542 and 704 bp respectively when genotyping h
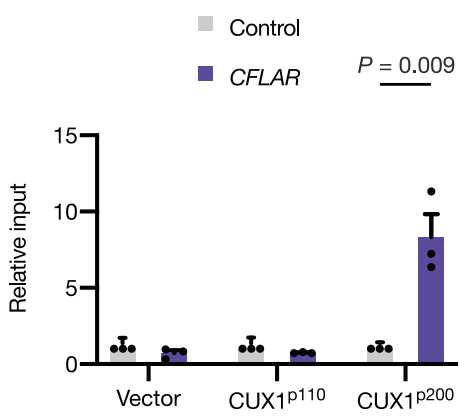

was performed with $\mathrm{P} 1, \mathrm{P} 2$ and $\mathrm{P} 3$ primers using the PCR parameters: $95^{\circ} \mathrm{C}$ for $15 \mathrm{~min} ; 35 \mathrm{cycles}$ of $95^{\circ} \mathrm{C}$ for $30 \mathrm{~s}, 58^{\circ} \mathrm{C}$ for $30 \mathrm{~s}, 72^{\circ} \mathrm{C}$ for $45 \mathrm{~s}$; and $72^{\circ} \mathrm{C}$ for $5 \mathrm{~min}$ for the final extension step. Vav-iCre mice were genotyped by PCR using VavF and VavR primers. Flt $3^{I T D}$ mice were genotyped using Flt314F and Flt314R primers. The genotypes of the mice were not blinded or randomized due to experimental design. Mice were maintained in $12 \mathrm{~h}$ light:12 h dark cycle environment with an ambient temperature of $21 \pm 2{ }^{\circ} \mathrm{C}$ and humidity of $55 \pm 10 \%$. All procedures were performed in accordance with UK Home Office regulations, UK Animals (Scientific Procedures) Act 1996 and with approval from the Animal Welfare Committee at the Wellcome Sanger Institute.

Human samples. Human leukemia and $\mathrm{CD} 34^{+}$cells were obtained with informed consent from individuals recruited onto the University of Cambridge Blood and Stem Cell Biobank UK REC-approved study (07/MRE05/44). Experiments using human cells were conducted with ethical approval from this study and the 
Fig. 7 CFLAR is directly repressed by CUX1. a Quantification of CFLAR protein levels in CUX1-/- U937 cells compared with wild-type. $\beta$-Actin was used as a loading control. The experiment was performed three times. b Expression of CYBB and CFLAR transcripts normalized to HPRT1 in CUX1-/- U937 cells compared with wild-type by qRT-PCR. Biological and technical triplicates were analyzed. c Immunoblot showing expression of biotinylated CUX1p100 and CUX1P200 proteins (top) in doxycycline-treated CUX1-/- U937 cells harboring the indicated constructs. The corresponding levels of CFLAR are also shown (bottom). $\beta$-Actin was used as a loading control. The experiment was performed three times. $\mathbf{d}$ Promoter-luciferase assays showing luciferase activity in HEK293T cells transfected with the indicated constructs and a $\sim \mathrm{kb}$ CFLAR promoter-luciferase reporter. Values were normalized to empty vector. Three experiments were performed with triplicate samples in each experiment. e Promoter-luciferase assays with deletion constructs of the CFLAR promoterluciferase reporter (large green arrow). Blue ovals, potential CUX1-binding sites. White oval, mutated potential CUX1-binding site. TSS, transcriptional start site (nominated +1 position). Three experiments were performed with triplicate samples in each experiment. $\mathbf{f}$ Sequence of potential CUX1-binding sites in CFLAR promoter closest to TSS. Locations of binding sites relative to TSS are shown. Consensus CUX1-binding site is shown (top). Red, exact consensus match. $\mathbf{g}$ Representative electrophoretic mobility shift assay with indicated components and proximal ( -132 to -122$)$ or distal ( -156 to -146$)$ radiolabeled, potential CUX1-binding dsDNA probes. The experiment was performed twice with similar results. $\mathbf{h}$ Chromatin immunoprecipitation assay followed by QRT-PCR for the CFLAR promoter region (purple) or distal control region (gray) in CUX1-/- U937 cells complemented with the indicated constructs as shown in c. Biological and technical duplicates were analyzed. All plots show mean + s.e.m. Two-tailed, unpaired t-test.

Wellcome Sanger Institute. Samples were linked anonymized with linkage information held by the study custodian.

Mouse pathology. Blood was collected from tail veins into ethylenediaminetetraacetic acid (EDTA)-coated tubes. Automated peripheral blood counts were obtained using a Vet abc machine (scil). Blood smears and bone marrow cytospins were stained with May-Grünwald Giemsa solutions (Sigma). Mouse tissues were fixed in formalin and embedded in paraffin. Blocks were cut into $5 \mu \mathrm{m}$ sections and mounted onto glass slides prior to staining with hematoxylin and eosin. For immunohistochemistry, slides were microwaved in $10 \mathrm{mM}$ citric acid $(\mathrm{pH} \mathrm{6.0)}$ to retrieve antigens. Endogenous peroxidases were quenched using hydrogen peroxide and staining was performed using anti-myeloperoxidase antibody (1:100, ab9535, Abcam) diluted in TBST for $1 \mathrm{~h}$ at room temperature. Signal detection was accomplished using an HRP/DAB (ABC) Detection IHC kit (Abcam, ab64264). Sections were counterstained with hematoxylin before dehydration and coverslip mounting. Slide images were captured using a Nanozoomer digital slide scanner (Hamamatsu)

Flow cytometry and antibodies. Bone marrow cells were obtained by flushing femurs and tibiae with FACS buffer (PBS supplemented with 2\% FBS). Red celldepleted single-cell suspensions were prepared by passing cells through a $70 \mu \mathrm{m}$ cell strainer and treatment with ACK lysing buffer (Lonza). Cells were treated with Fc Block (BD Biosciences) in cases where anti-CD16/32 staining was not performed. Cells were labeled with monoclonal antibodies or streptavidin-linked fluorophores suspended in FACS buffer for $30 \mathrm{~min}$ on ice. All flow cytometry reagents were purchased from eBioscience, BioLegend or BD Biosciences. For flow cytometric analysis of specific hematopoietic populations, lineage-positive cells were identified using Gr1 (RB6-8C5), CD11b (M1/70), Ter119 (TER119), B220 (RA3-6B2), CD4 (RM4-5), CD8 (53-6.7) and CD3 (145-2C11) antibodies conjugated to biotin used at 1:100 dilution. A Lineage Cell Depletion Kit (Miltenyi) was used to deplete lineage-positive cells prior to cell sorting. To reveal hematopoietic populations, cells were incubated with cocktails of the following antibodies used at 1:100 dilution: c-Kit (2B8), Scal (D7), CD150 (TC15-12F12.2), CD48 (HM48-1), CD34 (RAM34), CD16/32 (93), which were conjugated to PE, PercPCy5.5, PE-Cy7 or FITC. Biotin-conjugated antibodies were revealed using streptavidin conjugated to APC, APC-Cy7 or eFluor 450 used at 1:200 dilution. Live cells were identified by DAPI (Thermo) exclusion or Fixable Viability Dye eFluor 780 (eBioscience). Populations were defined as follows: LT-HSCs, CD150 ${ }^{+} \mathrm{CD} 48^{-}$ LSK; ST-HSC, CD150-CD48-LSK; MPP, CD150-CD48+ ${ }^{-}$LSK; GMPs, Lin-Sca-1 ${ }^{-}{ }^{-c K i t}$ ${ }^{+} \mathrm{CD} 34^{\text {hi }}{ }^{-} \mathrm{c} \gamma \mathrm{RII} / \mathrm{III}{ }^{\mathrm{hi}}$; CMPs, Lin-Sca- $1^{-}{ }^{-} \mathrm{CKit}{ }^{+} \mathrm{CD} 34^{\text {hi }} \mathrm{Fc} \gamma \mathrm{RII} / \mathrm{III}{ }^{\text {low; }}$ MEPs, Lin`Sca$1^{-}{ }^{-} \mathrm{Cit}^{+} \mathrm{CD} 34^{\text {low }} \mathrm{Fc} \gamma \mathrm{RII} / \mathrm{III}{ }^{\text {low. }}$. For lineage analysis, cells were stained with combinations of Gr1, Mac1, B220, CD4 and CD8 antibodies. For cell cycle analysis, cells were initially stained with antibodies to recognize live hematopoietic cell subpopulations before treatment with Cytofix/Cytoperm buffer kit (BD Biosciences), followed by Ki-67 (1:100, 16A8, 652406, BioLegend) and DAPI staining. Active caspase-3 staining was performed in a similar manner as cell cycle staining, but used anti-active caspase-3 Alexa Fluor 647 (1:50, 560626, BD Biosciences) and fixable viability dye to distinguish active caspase- 3 positive and dead cells respectively. An annexin-V apoptosis detection kit (BD Biosciences) was used to evaluate apoptosis according to the manufacturer's instructions. DAPI staining of ethanolfixed U937 CUX1-/- cells, was used to determine cell cycle distribution, whereas BioLegend antibodies to CD11b (ICRF44), CD14 (M5E2), CD34 (581) and CD33 (WM53) were used at 1:100 dilution to characterize the immunophenotype of these cells. Cell sorting was performed on a BD Influx machine (BD Biosciences). Flow cytometric data was acquired using Fortessa or LSR II instruments (BD Biosciences). Data were analyzed using FlowJo software (v10.0.8r1, Tree Star). Gating strategies to identify hematopoietic stem and progenitor cell populations are provided in Supplementary Fig. 11.
Bone marrow transplantation. For leukemia transplant assays, $2 \times 10^{5}$ red celldepleted bone marrow cells from Flt3 $3^{I T D}$ or $\mathrm{Cux}^{+/-} ; \mathrm{Flt3}{ }^{I T D}$ mice were injected into irradiated $\left(5.4 \mathrm{~Gy}\right.$, given as two split doses $4 \mathrm{~h}$ apart) CD45.1 $1^{+}$B6.SJLPtprc $^{a} P e p c^{b} /$ BoyJ mice along with $2 \times 10^{5}$ wild-type CD45.1 $1^{+}$bone marrow support cells. Transplanted animals were monitored for disease development by serial blood counts and daily examination. For in vivo Cflar knockdown competitive transplant experiments, $10^{5}$ Tet-pLKO puro shCflar-infected c-Kit ${ }^{+}$cells from Con, $\mathrm{Cu} x 1^{+-}$, Flt $3^{I T D}$ or $\mathrm{Cux}^{1^{+/-}}$Flt $3^{\text {ITD }} \mathrm{CD} 45.2^{+}$mice were injected with an equal number of $\mathrm{CD} 45.1^{+} \mathrm{CD} 45.2^{+}$wild-type bone marrow cells into lethally irradiated of CD45. $1^{+} \mathrm{CD} 45.2^{+}$recipients. Doxycycline chow containing $625 \mathrm{mg} / \mathrm{kg}$ doxycycline (TD.01306, Envigo) was given to the experimental cohort to induce Cflar knockdown following establishment of comparable engraftment, whereas the control groups were maintained on normal diet. Bone marrow donor CD45.2 chimerism was distinguished from CD45.1 $1^{+} \mathrm{CD} 45.2^{+}$support and recipient cells by flow cytometry two weeks later.

Immunoblotting. Cells were rinsed in PBS before lysis in RIPA buffer $(50 \mathrm{mM}$ Tris [pH 7.4], $150 \mathrm{mM} \mathrm{NaCl}, 1 \mathrm{mM}$ EDTA, $1 \%$ Triton X-100, $0.1 \%$ sodium deoxycholate, $0.1 \%$ SDS) supplemented with protease/phosphatase inhibitor cocktail (Cell Signaling Technology). Nuclear lysates were prepared according to standard methods. Samples were cleared by centrifugation at $16,000 \times g$ at $4{ }^{\circ} \mathrm{C}$ for $10 \mathrm{~min}$ and protein concentrations were determined using BCA protein assay reagent (Pierce). Equivalent amounts of protein were resolved on 4-12\% Bis-Tris or 3-8\% Tris-Acetate protein gels (NuPAGE) according to the manufacturer's instructions and transferred to nitrocellulose membranes (Amersham). Immunoblotting was performed according to standard procedures. The following primary antibodies used at 1:1,000 dilution were purchased from Cell Signaling Technology: antiHDAC1 (5356); anti-CFLAR (56343); anti- $\beta$-Actin (3700); anti-CASP8 (9746); mouse-specific anti-CASP8 (4927); anti-cleaved CASP8 (9496); mouse-specific anti-cleaved CASP8 (8592); anti-PARP (9542); anti-CASP3 (9662); anti-cleaved CASP3 (9661); anti-cleaved PARP (5625); anti-MLKL (37705); anti-p-MLKL ${ }^{\mathrm{S345}}$ (37333); anti-XIAP (2042); anti-NFkB p65 (8242); anti- IкBa (4814); anti-NFkB p100/p52 (4882); and anti-vinculin (13901). Other antibodies used at 1:1,000 dilution were: anti-CUX1 (ABE218, Millipore; 11733-1-AP, Proteintech); anti-myc (MA1-980, Invitrogen); anti-cIAP1/2 (MAB3400, Novus); and anti-Vinculin (SAB4200080, Sigma). HRP-linked streptavidin (1:1,000, 3999) and secondary antibodies (1:2,000, anti-rabbit IgG, 7074; 1:2,000, anti-mouse IgG, 7076) were from Cell Signaling Technology. Blots were treated with ECL reagent (Amersham) and signals visualized using an ImageQuant LAS 4000 machine (Amersham) or Xray film developed with a Compact X4 machine (Xograph). Band quantification was performed using ImageJ (v1.51, NIH).

Reverse phase protein array (RPPA). Logarithmically growing cells were collected and washed in PBS before freezing cell pellets at $-80^{\circ} \mathrm{C}$. Cell samples and arrays were processed by the RPPA Core at MD Anderson Cancer Center according to published procedures. Post-translationally modified proteins were normalized to total protein levels where possible. Description of sample processing array methods and data analysis are available on the facility's website. Three biological replicates per genotype were submitted for analysis.

Virus production and infection. Retroviral supernatants were produced by transfecting HEK293T cells with transfer and pCL-Ampho or pCL-Eco packaging plasmids. Tet-pLKO-puro, pLKO-GFP, pLKO-mCherry, pLKO.pig, pKLV2-EF1aCas9Bsd-W (Addgene, 68343), and pKLV2-based sgRNA lentiviral supernatants were produced by co-transfecting transfer, psPAX2 (Addgene, 12260) and pMD2.G (Addgene, 12259) plasmids into 293FT cells. Lipofectamine 3000 (Invitrogen) was used for transfections according to the manufacturer's instructions. Viral supernatants were collected $48 \mathrm{~h}$ later and frozen at $-80^{\circ} \mathrm{C}$. Cell lines were infected with 

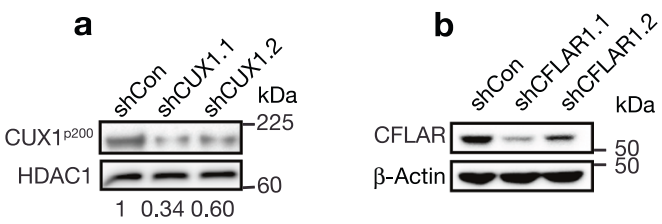

d

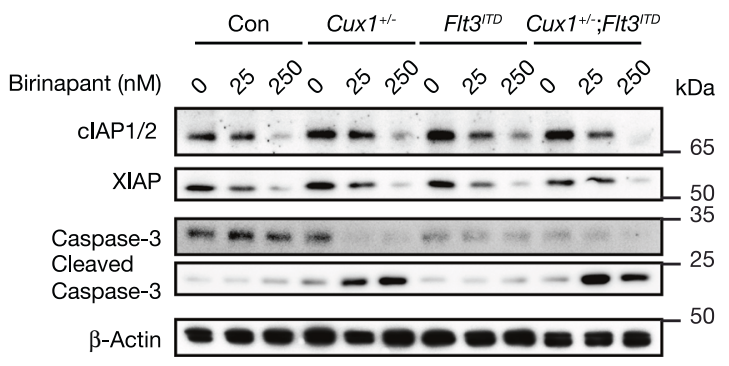

c

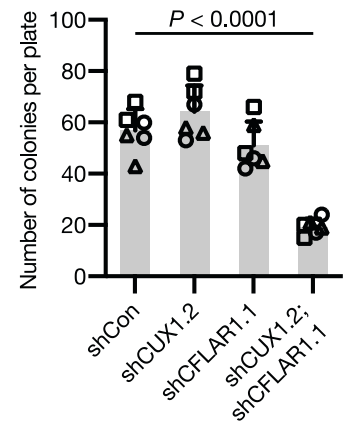

e
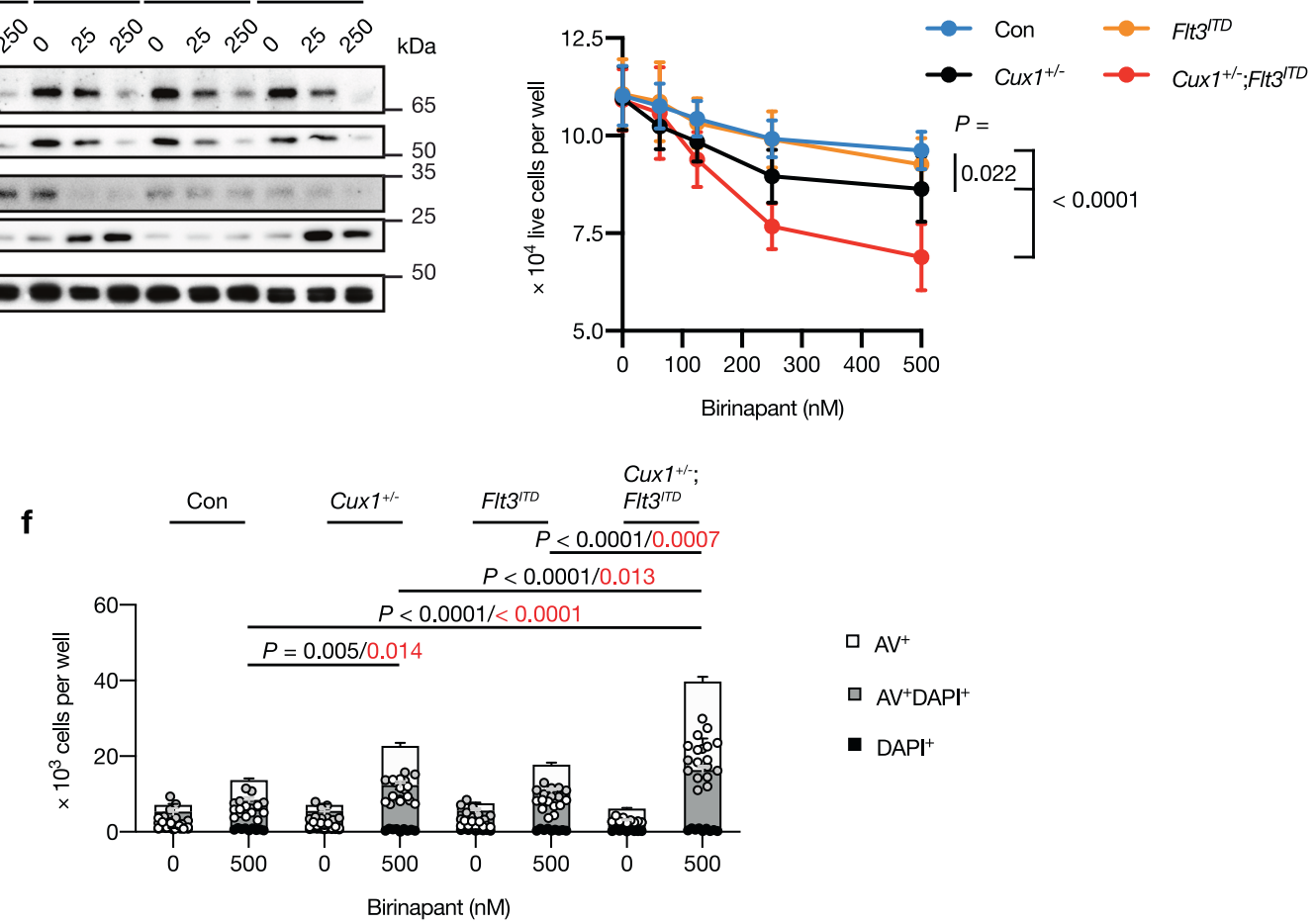

g

$95 \mathrm{~W}: 3 \times 10^{5}$ cells

$91 \mathrm{~W}: 3 \times 10^{5}$ cells

$91 \mathrm{~V}: 2 \times 10^{5}$ cells

$31 \mathrm{~A}: 1 \times 10^{5}$ cells

310: $1 \times 10^{5}$ cells
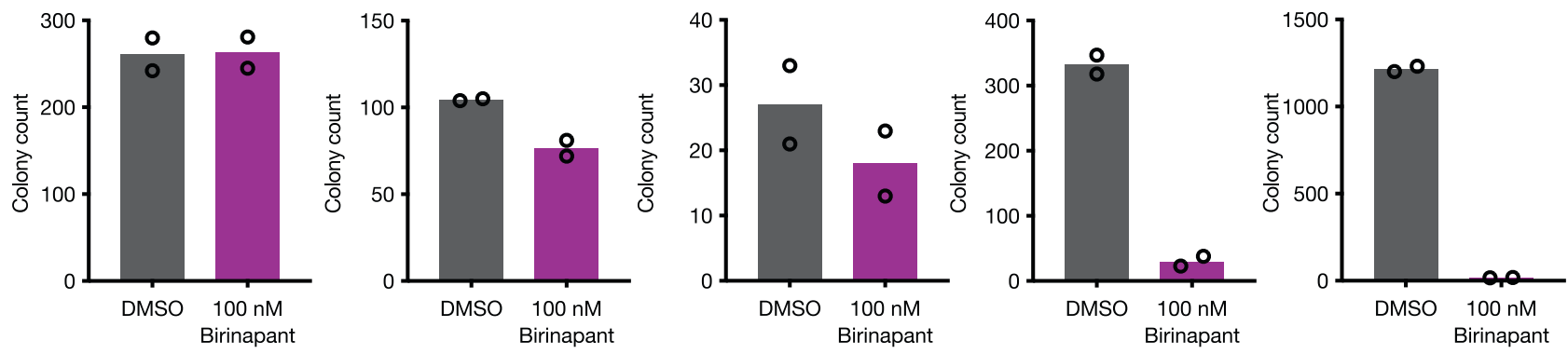

Normal karyotype cases

$-7 /$ del $(7 q)$ cases

filtered viral supernatants using cell growth media supplemented with $8 \mu \mathrm{g} / \mathrm{ml}$ polybrene (Sigma) for $12-16 \mathrm{~h}$. G418 $(1 \mu \mathrm{g} / \mathrm{ml}$, Gibco) was added $24 \mathrm{~h}$ later for MSCV-based infections, whereas puromycin (InvivoGen) was added to $1 \mu \mathrm{g} / \mathrm{ml}$ to select for lentivirus-transduced cells were required.

Mouse c-Kit ${ }^{+}$cells were isolated from hindlimb bone marrow using CD117 MicroBeads (Miltenyi) and resuspended in StemSpan SFEM media (STEMCELL Technologies) supplemented with glutamine, antibiotics and cytokines (mouse interleukin (IL)-3, $10 \mathrm{ng} / \mathrm{ml}$; mouse IL-6, $10 \mathrm{ng} / \mathrm{ml}$; and mouse stem cell factor (SCF), $25 \mathrm{ng} / \mathrm{ml}$; Peprotech). Cells were transduced by spinoculation at $800 \times g$ for $2 \mathrm{~h}$ at $32{ }^{\circ} \mathrm{C}$ with viral particles previously bound to RetroNectin-coated (Takara) six-well plates. Cells were incubated at $37^{\circ} \mathrm{C}$ in $5 \% \mathrm{CO}_{2}$ for a further $48 \mathrm{~h}$ before use in downstream experiments. Puromycin was added to $1 \mu \mathrm{g} / \mathrm{ml}$ to select for transduced cells where required. Doxycycline (Sigma) was added to $1 \mu \mathrm{g} / \mathrm{ml}$ to induce shRNA expression in methylcellulose assays.

Human $\mathrm{CD}_{3} 4^{+}$bone marrow cells from healthy donors were incubated for two days in StemPro-34 (Gibco) supplemented with StemPro Nutrient Supplement, glutamine, penicillin/streptomycin, human SCF $(50 \mathrm{ng} / \mu \mathrm{l})$, human thrombopoietin (150 ng/ $\mathrm{ll})$, human Flt3L (50 ng/ $\mu \mathrm{l})$ and human IL-6 $(50 \mathrm{ng} / \mu \mathrm{l})$ before virus transduction by spinoculation as above. Transduced cells were isolated by sorting for GFP and mCherry expression $48 \mathrm{~h}$ later prior to plating into methylcellulose media. 
Fig. 8 CUX1-haploinsufficient cells are sensitive to birinapant treatment. a, b Immunoblot showing knockdown of CUX1 (shCUX1.1 and shCUX1.2) (a) and CFLAR (shCFLAR1.1 and shCFLAR1.2) (b) using two independent shRNA vectors. A non-targeting control (shCon) was used as a knockdown control. HDAC1 or $\beta$-Actin were used as loading controls. The ratios of CUX1/HDAC1 levels normalized to shCon are shown below the blot in $\mathbf{a}$. The blot was performed twice. c Methylcellulose colony-forming assays using 500 human CD34+ cells transduced with the indicated shRNAs. Each shape represents data from an individual donor $(n=3)$. Cells were plated in duplicate and colonies containing more than 50 cells were scored. $\mathbf{d}$ Immunoblot showing the

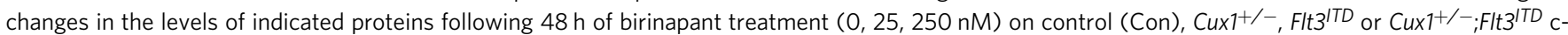
$\mathrm{Kit}^{+}$cells. $\beta$-Actin was used as a loading control. The experiment was performed twice. e Effect of birinapant treatment $(0-500 \mathrm{nM})$ on growth of $2 \times 10^{4}$ $\mathrm{c}-\mathrm{Kit}^{+}$cells from three mice of the indicated genotypes which were plated in triplicate. The number of live cells per well was determined by Annexin-V/ DAPI staining $48 \mathrm{~h}$ later. $\mathbf{f}$ Apoptosis assays using Annexin-V/DAPI staining of cells in $\mathbf{e}$. The total number of early- and late-apoptotic cells per well for each genotype was determined. Statistical significance was determined for birinapant-treated groups (black, $\mathrm{AV}^{+}$cells; red, $\mathrm{AV}+\mathrm{DAPI}+$ cells). $\mathbf{g}$ Bar plots of colony counts from methylcellulose colony-forming assays using $-7 / \operatorname{del}(7 q)$ or normal karyotype mononuclear cells from $A M L$ patients treated with $100 \mathrm{nM}$ birinapant or DMSO. The number of plated cells is shown above each graph. Cells were plated in duplicate for each patient. Colonies were counted 14 days after plating. Plots show mean \pm s.e.m. One-way ANOVA with Dunnett's test for multiple comparisons (c), two-way ANOVA with Tukey's test for multiple comparisons (e), one-way ANOVA with Tukey's test for multiple comparisons (f).

Methylcellulose colony assays. Murine bone marrow c-Kit ${ }^{+}$cells were resuspended in IMDM media (Gibco) and added to M3434 methylcellulose media (STEMCELL Technologies) before being dispensed into $35 \mathrm{~mm}$ dishes to obtain 500 cells/dish. Cells were incubated at $37{ }^{\circ} \mathrm{C}$ in $5 \% \mathrm{CO}_{2}$ for seven days before scoring colony numbers. Patient-derived hematopoietic and transduced human $\mathrm{CD} 34^{+}$cells were mixed with $\mathrm{H} 4435$ methylcellulose media (STEMCELL Technologies) and plated into $35 \mathrm{~mm}$ dishes with birinapant or drug vehicle as indicated (1-3 $\times 10^{5}$ plate for patient-derived cells; 500 cells/plate for human $\mathrm{CD} 34^{+}$cells). Plates were incubated for 13 days before staining with $0.1 \% \mathrm{p}$-iodonitrotetrazolium violet solution overnight. Plate images were captured and colonies were enumerated using Image software (v1.52b, NIH) or counted manually.

CRISPR/Cas9 drop-out screen. Wild-type and CUX1-/- U937 cells were transduced with pKLV2-EF1a-Cas9Bsd-W viral supernatant to generate stable Cas9-expressing cell lines after blasticidin (InvivoGen, $10 \mu \mathrm{g} / \mathrm{ml}$ ) selection. Cas9 activity was assessed with GFP reporter assays following transduction of Cas9expressing cells with pKLV2-U6gRNA5(gGFP)-PGKBFP2AGFP-W or pKLV2U6gRNA5(Empty)-PGKBFP2AGFP-W lentiviral supernatants. To achieve a 200fold library representation and a transduction efficiency of $\sim 30 \%$ as measured by BFP fluorescence (in order to avoid multiple sgRNAs per cell), $6.1 \times 10^{7}$ CUX1wild-type and $\mathrm{CUX1}^{-1-}$ Cas9-expressing U937 cells were transduced with the Human Improved Genome-wide Knockout CRISPR Library vl (Addgene, 67989), which contains 90,709 guides targeting 18,010 genes (3-5 sgRNAs per gene). The volume of viral supernatant required for each screen was calculated from preliminary titration experiments. Puromycin $(1 \mu \mathrm{g} / \mathrm{ml})$ was added two days after infection to select for sgRNA-infected cells and cultured for an additional 21 days (around 16 cell doublings in total), ensuring that $45 \times 10^{6}$ cells, representative of $\sim 500$ cells per sgRNA, were subcultured to maintain cell densities of $0.25-1.5 \times 10^{6}$ cells $/ \mathrm{ml}$. The sgRNAs from transfected CUX1-/-, CUX1-wild-type and the sgRNA plasmid library were amplified by PCR from DNA and sequenced as previously described ${ }^{60}$. Adapter-trimmed fastq sequencing reads were directly compared to a list of sgRNA sequences ${ }^{60}$ to quantify the abundance of each sgRNA in each sample. BAGEL v. $0.9^{61}$ was used to compute the log fold-change of each sgRNA in CUX1 $1^{-1-}$ and wild-type replicates compared with the plasmid library at timepoints up to day 21 after drug selection. BAGEL was used to calculate a Bayes Factor (BF) lethality score for each gene at a given timepoint. At the day 21 timepoint we computed a linear model of the gene BF for $\mathrm{CUX1}^{-1-}$ cell lines versus the gene BF for wild-type cell lines. The residuals of the linear model indicate the deviation of the gene-level lethality of the library in the CUX1-/- compared with the wild-type. Higher residual values suggest that knockout of the target gene in CUX1- $1^{--}$cells is significantly more lethal than in wild-type cells. The $\mathrm{Z}$ score of each residual was computed as Z_gene $=[$ residual(gene) - mean(all_residuals) $]$ / stddev (all_residuals).

Competitive sgRNA abundance validation assays. Wild-type or CUX1-/- U937 or THP-1 cells were infected with lentiviral supernatants to co-express individual sgRNAs targeting CFLAR or AIPL1 (a nonessential gene serving as a control), linked to BFP. The abundance of $\mathrm{BFP}^{+}$cells was determined by flow cytometry over a 20-21-day period in each case. Results were normalized to starting values and $\mathrm{BFP}^{+}$cell abundances in $\mathrm{CUX1} 1^{-/-}$cells were calculated relative to wild-type frequencies. Experiments were performed in triplicate.

Quantitative RT-PCR (qRT-PCR). Total RNA was extracted using RNeasy Mini or Micro kits (Qiagen) depending on cell input number. Genomic DNA was digested by including an on-column DNase step and RNA integrity was evaluated using a NanoDrop ND-1000 spectrophotometer (Thermo) or an RNA 6,000 Pico kit (Agilent). RNA was reversed transcribed using SuperScript VILO Master Mix (Invitrogen). Quantitative RT-PCR was performed using Taqman Fast Advanced
Master Mix (Applied Biosystems) on a QuantStudio 7 machine (Applied Biosystems). Gene expression was normalized to HPRT1 or Gapdh probe expression. Fold expression changes were calculated using the $\Delta \Delta \mathrm{Ct}$ method. Taqman probes: HPRT1, Hs99999909_m1; CYBB, Hs00166163_m1; CFLAR, Hs01116280_m1; Cflar, Mm01255578_m1; Gapdh, Mm99999915_g1.

Chromatin immunoprecipitation (ChIP). Doxycycline-inducible mCherry, CUX1 ${ }^{100}$ and CUX1 200 U937 cells were treated with $1 \%$ formaldehyde for $10 \mathrm{~min}$ at room temperature, followed by $0.125 \mathrm{M}$ glycine for a further $5 \mathrm{~min}$. Cells were washed in PBS and cell pellets were frozen at $-80^{\circ} \mathrm{C}$ prior to ChIP experiments. For ChIP, cells were resuspended in lysis buffer (10 mM Tris- $\mathrm{HCl}[\mathrm{pH} 8.0], 10 \mathrm{mM}$ $\mathrm{NaCl}, 0.2 \%$ IGEPAL CA-630, $1 \mathrm{mM}$ PMSF, cOmplete protease inhibitor [Roche]) for $10 \mathrm{~min}$ on ice. Nuclei were pelleted by centrifugation and lysed in nuclear buffer (50 mM Tris-HCl [pH 8.0], 10 mM EDTA, 1\% SDS, 1 mM PMSF, cOmplete protease inhibitor [Roche]). DNA was sheared with seven cycles $30 \mathrm{~s}$ on/off using a Bioruptor Pico device (diagenode). The soluble chromatin fraction was recovered and diluted with dilution buffer (20 mM Tris- $\mathrm{HCl}[\mathrm{pH} 8.0], 150 \mathrm{mM} \mathrm{NaCl}, 2 \mathrm{mM}$ EDTA, $1 \%$ Triton X-100, 0.01\% SDS, $1 \mathrm{mM}$ PMSF, cOmplete protease inhibitor [Roche]). Samples were incubated with Dynabeads MyOne Streptavidin T1 (Invitrogen), previously blocked with $1 \%$ fish gelatin (Sigma), overnight at $4{ }^{\circ} \mathrm{C}$. The following morning, beads were washed with $2 \%$ SDS, followed by wash 1 (50 mM HEPES, pH 7.5, $500 \mathrm{mM} \mathrm{NaCl}, 1 \mathrm{mM}$ EDTA, $1 \%$ Triton X-100, $0.1 \%$ deoxycholic acid), wash 2 (10 mM Tris-HCl, pH 8.0, $250 \mathrm{mM} \mathrm{LiCl,} 1 \mathrm{mM}$ EDTA $0.5 \%$ IGEPAL CA-630, $0.5 \%$ deoxycholic acid) and TE buffer (10 mM Tris- $\mathrm{HCl}$, $\mathrm{pH} 7.5,1 \mathrm{mM}$ EDTA). DNA was eluted from beads using elution buffer $(50 \mathrm{mM}$ Tris- $\mathrm{HCl}, \mathrm{pH} 8.0,1 \% \mathrm{SDS}, 10 \mathrm{mM}$ EDTA) at $65^{\circ} \mathrm{C}$ overnight with agitation using a ThermoMixer (Eppendorf). Samples were treated with RNase A (Invitrogen) and proteinase $\mathrm{K}$ (Ambion) before recovery of genomic DNA using phenol chloroform. ChIP qRT-PCR was performed using PowerUp SYBR Green Master Mix (Applied Biosystems) on a QuantStudio 7 machine (Applied Biosystems). The percentage input method was used to calculate DNA recovery of specific genomic loci. Primers corresponding to the CFLAR promoter were CFLARChF1 and CFLARChR1. Primers annealing to a distal control genomic region were CFLAR_CHIPconF and CFLAR_CHIPconR.

Proliferation and drug treatment assays. $1 \times 10^{4} \mathrm{U} 937 \mathrm{CUX1} 1^{-/-}$or wild-type cells were dispensed into 96 well plates in triplicate. Cytarabine (Apexbio) was dissolved in water. Drug or vehicle were added to cells to achieve the desired concentrations as indicated. Cells were incubated for $48 \mathrm{~h}$ before cell viability was determined using CellTiter $96 \mathrm{AQ}_{\text {ueous }}$ One reagent (Promega). For birinapant treatment of murine $\mathrm{cKit}^{+}$cells, $2 \times 10^{4}$ cells were plated in 96 well plates containing RPMI supplemented with 10\% FBS, glutamine, antibiotics and cytokines (mouse IL-3, $10 \mathrm{ng} / \mathrm{ml}$; mouse IL-6, $10 \mathrm{ng} / \mathrm{ml}$; and mouse SCF, $25 \mathrm{ng} / \mathrm{ml}$; Peprotech) in triplicate from three mice per genotype. Birinapant (Selleckchem) or DMSO vehicle was added to achieve desired concentrations and a final well volume of $200 \mu \mathrm{l}$ before incubating for a further $48 \mathrm{~h}$. Proliferation assays using murine cKit $^{+}$cells infected with Cflar shRNA vectors were performed similarly using $2 \times$ $10^{4}$ cells plated in 96 well plates in triplicate from two mice per genotype with doxycycline (Sigma) or drug vehicle added to $0.1 \mu \mathrm{g} / \mathrm{ml}$. Apoptosis was determined by Annexin-V and DAPI staining. Necrostatin-1 (20 $\mu \mathrm{M}$, Sigma) or zVAD (10 $\mu \mathrm{M}$, Selleckchem) was added at the same time as birinapant or doxycycline where indicated in the text.

Plasmids, shRNAs and siRNA. Lentiviral transfer plasmids expressing sgRNAs targeting CFLAR, and AIPL1 genes were generated by cloning annealed sgRNA oligonucleotides (CFLAR1_For, CFLAR1_Rev; and AIPL1_For, AIPL1_Rev), into the BbsI site of pKLV-U6gRNA(BbsI)-PGKpuro2ABFP (Addgene, 50946), leading to pKLV-U6gRNA(CFLAR1)-PGKpuro2ABFP and pKLV-U6gRNA(AIPL1)- 
PGKpuro2ABFP plasmids respectively. For CRISPR/Cas9-resistant CFLAR complementation experiments, CFLAR2_For and CFLAR2_Rev oligonucleotides were annealed and cloned into of pKLV-U6gRNA(BbsI)-PGKpuro2ABFP, leading to pKLV-U6gRNA(CFLAR2)-PGKpuro2ABFP. Lentiviral sgRNA transfer plasmids targeting CUX1 and ACCSL genes were generated by cloning annealed sgRNA oligonucleotides (CUX1crsp9_For, CUX1crsp9_Rev; and ACCSL_For, ACCSL_Rev), into the BbsI site of pKLV2-U6gRNA5(BbsI)-PGKpuro2AmCherryW (Addgene, 67977), leading to pKLV2-U6gRNA5(CUX1)-PGKpuro2AmCherry$\mathrm{W}$ and pKLV2-U6gRNA5(ACCSL)-PGKpuro2AmCherry-W plasmids respectively. The non-essential AIPL1 and ACCSL genes were used as control sgRNA targets for CRISPR/Cas9 experiments. For CUX1 ablation in U937 cells, two sgRNA sequences, annealed CUX1crsp8 (CUX1crsp8_For and CUX1crsp8_Rev) and CUX1crsp9 (CUX1crsp9_For and CUX1crsp9_Rev) oligonucleotides were cloned into pX458 (Addgene, 48138) which target exon 18 of CUX1, to generate sgRNA-expressing vectors pX458 CUX1crsp8 and pX458 CUX1crsp9 respectively. Relevant portions of all plasmids were verified by DNA sequencing or restriction digests. Enzymes were purchased from New England Biolabs.

To create doxycycline-regulatable vectors expressing epitope-tagged CUX1p110 or CUX1 ${ }^{200}$ that are substrates for biotin holoenzyme synthetase (BirA), CUX1 $1^{110}$ or CUX1 $1^{200}$ coding sequences were subcloned from $\mathrm{pXI}$ mycCUX1 ${ }^{\mathrm{p} 110}$-HA and pXJ myc-CUX1 ${ }^{\mathrm{p} 200}$-HA into pKS Bio-FLAG so that CUX1 isoforms were $\mathrm{N}$ - and C-terminally tagged with biotinylatable peptides and HA tags respectively. Epitope-tagged CUX1 proteins or mCherry were subsequently subcloned downstream of a tetracycline response element (TRE) in PB BirA-P2ArtTA-EGFP-TRE which also harbors: BirA, rtTA and EGFP coding sequences separated by P2A motifs under the control of a CAGGS promoter; and flanking Piggybac inverted terminal repeats. PB BirA-P2A-rtTA-EGFP-TRE mCherry, PB BirA-P2A-rtTA-EGFP-TRE CUX1p110 and PB BirA-P2A-rtTA-EGFP-TRE CUX1 ${ }^{\mathrm{P} 200}$ plasmids were verified by sequencing.

For human CFLAR expression, an IRES-EGFP cassette from pIRES2-EGFP (Clontech) was firstly cloned downstream of the neomycin-resistance gene in MSCVneo (Clontech) to create MSCVneo IRES-EGFP. The CFLAR coding sequence with a C-terminal V5 tag was cloned into MSCVneo IRES-EGFP, leading to MSCVneo IRES-EGFP CFLAR-V5. For mouse Cflar expression, the mouse Cflar ORF was amplified from IMAGE clone 4460554 using mCflarHpaF and mCflarSalR by PCR. The PCR product was cut with HpaI and SalI and cloned into MSCV IRES GFP which had been cut with the same enzymes, creating MSCV mCflar IRES EGFP.

To construct CFLAR promoter-luciferase vectors, the genomic region upstream of the CFLAR transcriptional start site was amplified by PCR using CFLARpr_KpnRVFor and CFLARpr_XhNhRev. The PCR product was digested with NheI, blunted with Klenow and cut subsequently with KpnI prior to cloning in pGL4.10[luc2] (Promega), which had been digested with HindII, treated with Klenow and cut subsequently with KpnI, thereby creating pGL4.10-

CFLARprom $^{\mathrm{WT}}$. CFLAR promoter-luciferase deletion constructs were made as follows from pGL4.10-CFLARprom ${ }^{W T}$ : pGL4.10-CFLARprom ${ }^{\Delta 1}$, EcoRV and BglII digest; pGL4.10-CFLARprom ${ }^{\Delta 2}$, HpaI-XhoI fragment was cloned into EcoRV and XhoI-digested pGL4.10-CFLARprom WT; pGL4.10-CFLARprom ${ }^{\Delta 3}$, EcoRV and HindIII digest; pGL4.10-CFLARprom ${ }^{\Delta 4}$, HindIII and XhoI digest. DNA ends were blunted with Klenow before religating vectors. pGL4.10-CFLARprom ${ }^{\Delta 5}$ was created from pGL4.10-CFLARprom ${ }^{\Delta 1}$ by KpnI and EcoRI digestion before Klenow treatment and religation. The putative CUX1-binding sites in pGL4.10CFLARprom $^{\Delta 5}$ was mutated by site-directed mutagenesis with Phusion DNA polymerase using CFLAR_mutF and CFLAR_mutR, leading to pGL4.10CFLARprom $^{\triangle 5 \mathrm{MUT}}$. To create doxycycline-regulatable Cflar shRNA vectors, two pairs of Cflar-targeting oligonucleotides (Cflar_sh1For, Cflar_sh1Rev; and Cflar_sh2For, Cflar_sh2Rev) were annealed and cloned into Tet-pLKO-puro (Addgene, 21915). For CUX1 shRNA knockdown in U937 and THP-1 cells, pLKO. pig shCon and pLKO.pig shCUX1 were used. ${ }^{18}$ To create CUX1 and CFLAR shRNA vectors for human $\mathrm{CD} 34^{+}$experiments, which co-express GFP or mCherry, two pairs of CUX1-, CFLAR- or control-targeting oligonucleotides (CUX1.1: CUXsh1F/ CUXsh1R, CUX1.2: CUX1sh2F/CUXsh2R; CFLAR1.2: CFLARshf1/CFLARshf2, CFLAR1.2: CFLARshR1/CFLARshR2; Control: ConF/R) were annealed and cloned into pLKO-GFP or pLKO-mCherry in which the puromycin resistance gene was replaced by the relevant fluorescent protein-coding sequence.

CFLAR promoter-luciferase assay. Promoter-luciferase assays were conducted using HEK293T cells as previously described ${ }^{18}$. HEK293T cells were transfected with Lipofectamine 2000 (Invitrogen) and luciferase activity was determined 24 later. Triplicate transfections were performed in each of three experiments. Data for each promoter construct were normalized to signals derived from pXJ42 empty vector controls.

Electrophoretic mobility shift assay. Nuclear lysates were prepared from HEK293T cells transfected with pXJ42 or pXJ myc-CUX1 ${ }^{200}-\mathrm{HA}$ vectors using lipofectamine 2000 (Invitrogen) according the manufacturer's instructions. Complementary oligonucleotides CFLAR_emsaF1 and CFLAR_emsaR1 representing putative -132 to -122 CUX1-binding sites were annealed and end-labeled using [ $\gamma$ $\left.{ }^{32} \mathrm{P}\right]$ ATP (Perkin Elmer) with T4 polynucleotide kinase (New England Biolabs). Labeled double-stranded DNA probes were prepared in the same way using
CFLAR_emsaF2 and CFLAR_emsaR2 oligonucleotides corresponding to potential -156 to -146 CUX1-binding sites. Unincorporated nucleotides were removed using illustra MicroSpin G-25 columns (GE Healthcare) according to the manufacturer's instructions. Binding reactions were performed by mixing $2 \mu \mathrm{g}$ nuclear lysate in $20 \mu \mathrm{l}$ final volume containing binding buffer [10 $\mathrm{mM}$ Tris ( $\mathrm{pH} 7.5), 25 \mathrm{mM} \mathrm{NaCl}$, $1 \mathrm{mM} \mathrm{MgCl} 2,5 \mathrm{mM}$ EDTA (pH 8.0), $1 \mathrm{mM}$ DTT and 5\% glycerol], $100 \mathrm{ng}$ poly (dI$\mathrm{dC}$ ) (Thermo), $3 \mu \mathrm{g}$ bovine serum albumin (Sigma) and $0.2 \mathrm{pmol}$ radiolabeled probe for $15 \mathrm{~min}$ at room temperature. Cold probe competition assays were performed by adding 10-fold molar excess of unlabeled DNA probe $10 \mathrm{~min}$ prior to adding the radiolabeled probe. Antibody inhibition assays were performed by preincubating nuclear lysates with normal mouse IgG (12-371, Upstate) or anti-myc antibody (05724, Upstate). Reactions were separated on 6\% DNA retardation gels (Novex) in $0.5 \times$ TBE buffer at $80 \mathrm{~V}$. Gels were dried and exposed to Hyperfilm MP films (Amersham), which were developed using a Compact X4 machine (Xograph).

Cell culture. THP-1 cells were sourced from the Sanger Cell Lines Project (https:// cancer.sanger.ac.uk/cell_lines), whereas U937 cells originated from the European Collection of Authenticated Cell Cultures. Cells were authenticated by short tandem repeat genotyping and verified to be free from mycoplasma contamination. Cells were grown at $37^{\circ} \mathrm{C}$ in $5 \% \mathrm{CO}_{2}$ using RPMI media supplemented with $10 \%$ fetal bovine serum (FBS, Gibco), glutamine, penicillin and streptomycin. HEK293T and 293FT (Invitrogen) cells were grown in DMEM media with 10\% FBS, glutamine, penicillin and streptomycin.

CUX1 knockout and CUX1-tagged cell line generation. U937 CUX1-1- cells were generated by electroporating $2.5 \mu \mathrm{g}$ of pX458 CUX1crsp8 and pX458 CUX1crsp9 plasmids (which express Cas9, an sgRNA targeting CUX1 exon 18 and EGFP) into $5 \times 10^{5}$ cells in a $100 \mu \mathrm{l}$ Neon tip using a Neon transfection system (pulse voltage $1400 \mathrm{~V}$, pulse width $10 \mathrm{~ms}$, pulse number 3) according to the manufacturer's instructions (Thermo). Forty-eight hours later, single $\mathrm{EGFP}^{+}$cells were isolated by flow cytometric sorting into 96 -well plates and cultured. CUX1-1clones were identified by PCR genotyping and DNA sequencing of CUX1 exon 18 using primers hCUXsurvF18 and hCUXsurvR18, followed by immunoblotting for CUX1 protein.

Doxycycline-regulatable mCherry, CUX1 ${ }^{1110}$ and CUX1 ${ }^{200}$ expression in U937 CUX1 $1^{-1-}$ cells was achieved by electroporating $2.5 \mu \mathrm{g}$ pCMV-hyPBase along with $2.5 \mu$ g PB BirA-P2A-rtTA-EGFP-TRE mCherry, PB BirA-P2A-rtTA-EGFPTRE CUX1 110 or PB BirA-P2A-rtTA-EGFP-TRE CUX1 ${ }^{200}$ into U937 CUX1 ${ }^{-1-}$ cells using a Neon transfection system. $\mathrm{EGFP}^{+}$cells were isolated by flow cytometric sorting and expanded. Protein expression was induced by adding doxycycline $(0.1 \mu \mathrm{g} / \mathrm{ml})$ to the growth media $24-36 \mathrm{~h}$ before cell harvest.

To generate THP-1 CUX1 $1^{-1-}$ and control sgRNA-expressing cell lines, THP-1 cells were first infected with Cas9-expressing pKLV2-EF1a-Cas9Bsd-W lentivirus and selected with blasticidin. THP-1:Cas9 cells were subsequently infected with lentiviral supernatants produced using pKLV2-U6gRNA5(CUX1)-

PGKpuro2AmCherry-W or pKLV2-U6gRNA5(ACCSL)-PGKpuro2AmCherry-W transfer plasmids and selected with puromycin.

RNA sequencing. Total RNA was extracted from sorted LSK mouse cells using RNeasy Micro kits with on column DNA digestion (Qiagen). A SMART-Seq v4 Ultra Low Input RNA kit (Clontech) was used to synthesize cDNA libraries from LSK cells. Total RNA samples were evaluated using an RNA 6,000 Pico kit (Agilent). Indexed sequencing libraries from LSK cDNA samples were prepared using a Nextera XT DNA library preparation kit (Illumina). Libraries were pooled and sequenced using an Illumina HiSeq 2000 system to produce 75 bp paired-end reads for LSK cells. For mouse transcriptome data analysis, adapter-trimmed sequences were mapped to the GRCm38 assembly with STAR (v2.5.0 ${ }^{62}$. Gene annotations were from Ensembl release 84. Gene-level quantification was performed using HTSeq (v.0.7.2) (ref. 63). Differential gene expression between control and experimental samples was determined using DESeq2 package $^{64}$ (v.1.24.0). GSEA (v4.0.3) was performed using curated gene sets from the Molecular Signatures Database and studies relevant to hematopoietic self-renewal and differentiation signatures ${ }^{65}$. DAVID (v6.8) was used for functional gene annotation ${ }^{66}$.

Statistical analyses. Two-tailed, unpaired $t$-tests or Mann-Whitney tests were performed to assess statistical significance in pairwise comparisons. Log-rank (Mantel-Cox) tests were performed on survival curves to determine significant differences. One-way or two-way ANOVA with Tukey's or Dunnett's tests were used for multiple group comparisons.

Reporting summary. Further information on research design is available in the Nature Research Reporting Summary linked to this article.

\section{Data availability}

The mouse LSK RNA-sequencing data for this study have been deposited in the European Nucleotide Archive (ENA) at EMBL-EBI under accession number P1. Molecular Signatures Database v7.2 is available through GSEA website (https://www. gsea-msigdb.org/gsea/msigdb/index.jsp). The source data underlying Figs. 1-8 and 
Supplementary Figures are provided as a Source Data file. All the other data supporting the findings of this study are available within the article and its supplementary information files and from the corresponding author upon reasonable request. Source data are provided with this paper.

Received: 20 January 2020; Accepted: 24 March 2021; Published online: 30 April 2021

\section{References}

1. Knudson, A. G. Jr. Mutation and cancer: statistical study of retinoblastoma Proc. Natl Acad. Sci. USA 68, 820-823 (1971).

2. Vaquerizas, J. M., Kummerfeld, S. K., Teichmann, S. A. \& Luscombe, N. M. A census of human transcription factors: function, expression and evolution. Nat. Rev. Genet 10, 252-263 (2009).

3. Sondka, Z. et al. The COSMIC Cancer Gene Census: describing genetic dysfunction across all human cancers. Nat. Rev. Cancer 18, 696-705 (2018).

4. Inoue, K. \& Fry, E. A. Haploinsufficient tumor suppressor genes. Adv. Med Biol. 118, 83-122 (2017).

5. Bhagwat, A. S. \& Vakoc, C. R. Targeting transcription factors in cancer. Trends Cancer 1, 53-65 (2015).

6. Solimini, N. L., Luo, J. \& Elledge, S. J. Non-oncogene addiction and the stress phenotype of cancer cells. Cell 130, 986-988 (2007).

7. Nagel, R., Semenova, E. A. \& Berns, A. Drugging the addict: non-oncogene addiction as a target for cancer therapy. EMBO Rep. 17, 1516-1531 (2016).

8. Luo, J. et al. A genome-wide RNAi screen identifies multiple synthetic lethal interactions with the Ras oncogene. Cell 137, 835-848 (2009).

9. Son, J. et al. Glutamine supports pancreatic cancer growth through a KRASregulated metabolic pathway. Nature 496, 101-105 (2013).

10. Murga, M. et al. Exploiting oncogene-induced replicative stress for the selective killing of Myc-driven tumors. Nat. Struct. Mol. Biol. 18, 1331-1335 (2011).

11. Lee, A. C. et al. Ras proteins induce senescence by altering the intracellular levels of reactive oxygen species. J. Biol. Chem. 274, 7936-7940 (1999).

12. Vafa, O. et al. c-Myc can induce DNA damage, increase reactive oxygen species, and mitigate $\mathrm{p} 53$ function: a mechanism for oncogene-induced genetic instability. Mol. Cell 9, 1031-1044 (2002).

13. Kaplon, J. et al. A key role for mitochondrial gatekeeper pyruvate dehydrogenase in oncogene-induced senescence. Nature 498, 109-112 (2013).

14. Dominguez-Sola, D. et al. Non-transcriptional control of DNA replication by c-Myc. Nature 448, 445-451 (2007).

15. Greenberg, P. L. et al. Revised international prognostic scoring system for myelodysplastic syndromes. Blood 120, 2454-2465 (2012).

16. Grimwade, D. et al. Refinement of cytogenetic classification in acute myeloid leukemia: determination of prognostic significance of rare recurring chromosomal abnormalities among 5876 younger adult patients treated in the United Kingdom Medical Research Council trials. Blood 116, 354-365 (2010).

17. Hosono, N. et al. Recurrent genetic defects on chromosome $7 \mathrm{q}$ in myeloid neoplasms. Leukemia 28, 1348-1351 (2014).

18. Wong, C. C. et al. Inactivating CUX1 mutations promote tumorigenesis. Nat. Genet 46, 33-38 (2014).

19. Aly, M. et al. Distinct clinical and biological implications of CUX1 in myeloid neoplasms. Blood Adv. 3, 2164-2178 (2019).

20. Patel, J. P. et al. Prognostic relevance of integrated genetic profiling in acute myeloid leukemia. N. Engl. J. Med 366, 1079-1089 (2012).

21. McNerney, M. E. et al. CUX1 is a haploinsufficient tumor suppressor gene on chromosome 7 frequently inactivated in acute myeloid leukemia. Blood 121, 975-983 (2013).

22. Hulea, L. \& Nepveu, A. CUX1 transcription factors: from biochemical activities and cell-based assays to mouse models and human diseases. Gene 497, 18-26 (2012).

23. He, X. et al. PIK3IP1, a negative regulator of PI3K, suppresses the development of hepatocellular carcinoma. Cancer Res 68, 5591-5598 (2008).

24. An, N. et al. Gene dosage effect of CUX1 in a murine model disrupts HSC homeostasis and controls the severity and mortality of MDS. Blood 131, 2682-2697 (2018).

25. McNerney, M. E. et al. The spectrum of somatic mutations in high-risk acute myeloid leukaemia with -7/del(7q). Br. J. Haematol. 166, 550-556 (2014)

26. Tyner, J. W. et al. Functional genomic landscape of acute myeloid leukaemia. Nature 562, 526-531 (2018).

27. Papaemmanuil, E. et al. Genomic classification and prognosis in acute myeloid leukemia. N. Engl. J. Med 374, 2209-2221 (2016).

28. Igney, F. H. \& Krammer, P. H. Death and anti-death: tumour resistance to apoptosis. Nat. Rev. Cancer 2, 277-288 (2002).

29. Hanahan, D. \& Weinberg, R. A. Hallmarks of cancer: the next generation. Cell 144, 646-674 (2011)
30. Irmler, M. et al. Inhibition of death receptor signals by cellular FLIP. Nature 388, 190-195 (1997).

31. Cho, Y. S. et al. Phosphorylation-driven assembly of the RIP1-RIP3 complex regulates programmed necrosis and virus-induced inflammation. Cell 137, 1112-1123 (2009)

32. He, S. et al. Receptor interacting protein kinase- 3 determines cellular necrotic response to TNF-alpha. Cell 137, 1100-1111 (2009).

33. Sun, L. et al. Mixed lineage kinase domain-like protein mediates necrosis signaling downstream of RIP3 kinase. Cell 148, 213-227 (2012).

34. Zhang, D. W. et al. RIP3, an energy metabolism regulator that switches TNFinduced cell death from apoptosis to necrosis. Science 325, 332-336 (2009).

35. Zhao, J. et al. Mixed lineage kinase domain-like is a key receptor interacting protein 3 downstream component of TNF-induced necrosis. Proc. Natl Acad. Sci. USA 109, 5322-5327 (2012).

36. Feoktistova, M. et al. cIAPs block Ripoptosome formation, a RIP1/caspase- 8 containing intracellular cell death complex differentially regulated by cFLIP isoforms. Mol. Cell 43, 449-463 (2011).

37. Ko, M. et al. Modulation of TET2 expression and 5-methylcytosine oxidation by the CXXC domain protein IDAX. Nature 497, 122-126 (2013).

38. Traver, D., Akashi, K., Weissman, I. L. \& Lagasse, E. Mice defective in two apoptosis pathways in the myeloid lineage develop acute myeloblastic leukemia. Immunity 9, 47-57 (1998).

39. Chang, D. W. et al. c-FLIP(L) is a dual function regulator for caspase- 8 activation and CD95-mediated apoptosis. EMBO J. 21, 3704-3714 (2002).

40. Lee, B. H. et al. FLT3 mutations confer enhanced proliferation and survival properties to multipotent progenitors in a murine model of chronic myelomonocytic leukemia. Cancer Cell 12, 367-380 (2007).

41. Hartmann, L. et al. Myeloid malignancies with isolated $7 \mathrm{q}$ deletion can be further characterized by their accompanying molecular mutations. Genes Chromosomes Cancer 58, 698-704 (2019).

42. Chu, S. H. et al. FLT3-ITD knockin impairs hematopoietic stem cell quiescence/homeostasis, leading to myeloproliferative neoplasm. Cell Stem Cell 11, 346-358 (2012).

43. Greenblatt, S. et al. Knock-in of a FLT3/ITD mutation cooperates with a NUP98-HOXD13 fusion to generate acute myeloid leukemia in a mouse model. Blood 119, 2883-2894 (2012).

44. Whitman, S. P. et al. Absence of the wild-type allele predicts poor prognosis in adult de novo acute myeloid leukemia with normal cytogenetics and the internal tandem duplication of FLT3: a cancer and leukemia group B study. Cancer Res. 61, 7233-7239 (2001).

45. Kiel, M. J. et al. SLAM family receptors distinguish hematopoietic stem and progenitor cells and reveal endothelial niches for stem cells. Cell 121, 1109-1121 (2005).

46. Socolovsky, M. et al. Ineffective erythropoiesis in Stat5a(-/-)5b(-/-) mice due to decreased survival of early erythroblasts. Blood 98, 3261-3273 (2001).

47. Barretina, J. et al. The Cancer Cell Line Encyclopedia enables predictive modelling of anticancer drug sensitivity. Nature 483, 603-607 (2012).

48. Lievens, P. M. J., Donady, J. J., Tufarelli, C. \& Neufeld, E. J. Repressor activity of CCAAT displacement protein in HL-60 myeloid leukemia cells. J. Biol. Chem. 270, 12745-12750 (1995).

49. Fulda, S., Vucic, D. \& Targeting, I. A. P. proteins for therapeutic intervention in cancer. Nat. Rev. Drug Disco. 11, 109-124 (2012).

50. McLornan, D. et al. Prognostic and therapeutic relevance of c-FLIP in acute myeloid leukaemia. Br. J. Haematol. 160, 188-198 (2013).

51. Abedini, M. R., Muller, E. J., Bergeron, R., Gray, D. A. \& Tsang, B. K. Akt promotes chemoresistance in human ovarian cancer cells by modulating cisplatin-induced, p53-dependent ubiquitination of FLICE-like inhibitory protein. Oncogene 29, 11-25 (2010).

52. Longley, D. B. et al. c-FLIP inhibits chemotherapy-induced colorectal cancer cell death. Oncogene 25, 838-848 (2006).

53. McCourt, C. et al. Elevation of c-FLIP in castrate-resistant prostate cancer antagonizes therapeutic response to androgen receptor-targeted therapy. Clin. Cancer Res 18, 3822-3833 (2012).

54. Ripka S., et al. CUX1: target of Akt signalling and mediator of resistance to apoptosis in pancreatic cancer. gutbmjcom.

55. Arthur, D. C. et al. The clinical significance of karyotype in acute myelogenous leukemia. Cancer Genet Cytogenet 40, 203-216 (1989).

56. Schiffer, C. A., Lee, E. J., Tomiyasu, T., Wiernik, P. H. \& Testa, J. R. Prognostic impact of cytogenetic abnormalities in patients with de novo acute nonlymphocytic leukemia. Blood 73, 263-270 (1989).

57. Humphreys, L., Espona-Fiedler, M. \& Longley, D. B. FLIP as a therapeutic target in cancer. FEBS J. 285, 4104-4123 (2018).

58. Carter, B. Z. et al. Small-molecule XIAP inhibitors derepress downstream effector caspases and induce apoptosis of acute myeloid leukemia cells. Blood 105, 4043-4050 (2005).

59. Donnellan, W. B. et al. A phase 2 study of azacitidine (5-AZA) with or without birinapant in subjects with higher risk myelodysplastic syndrome (MDS) or 
chronic myelomonocytic leukemia (CMML). J. Clin. Oncol. 34, 7060-7060 (2016).

60. Tzelepis, K. et al. A CRISPR dropout screen identifies genetic vulnerabilities and therapeutic targets in acute myeloid leukemia. Cell Rep. 17, 1193-1205 (2016).

61. Hart, T. \& Moffat, J. BAGEL: a computational framework for identifying essential genes from pooled library screens. BMC Bioinformatics 17, 164 (2016).

62. Dobin, A. et al. STAR: ultrafast universal RNA-seq aligner. Bioinformatics 29, 15-21 (2013).

63. Anders, S., Pyl, P. T. \& Huber, W. HTSeq-a Python framework to work with high-throughput sequencing data. Bioinformatics 31, 166-169 (2015).

64. Love, M. I., Huber, W. \& Anders, S. Moderated estimation of fold change and dispersion for RNA-seq data with DESeq2. Genome Biol. 15, 550 (2014).

65. Park, S. M. et al. Musashi-2 controls cell fate, lineage bias, and TGF-beta signaling in HSCs. J. Exp. Med 211, 71-87 (2014).

66. Huang da, W., Sherman, B. T. \& Lempicki, R. A. Systematic and integrative analysis of large gene lists using DAVID bioinformatics resources. Nat. Protoc. 4, 44-57 (2009). 01/20/2020; 03/24/2021.

\section{Acknowledgements}

This work was funded by a Wellcome Intermediate Fellowship (Grant Number: 105914/Z/ 14/Z) to C.C.W. and the Kay Kendall Leukemia Fund. D.J.A. is supported by Cancer Research UK, Wellcome and an ERC Combat Cancer Programme award. The plasmids pXJ42, pXJ myc-CUX1 ${ }^{110}$-HA and pXJ myc-CUX1 ${ }^{200}$-HA were kind gifts from A. Nepveu, whereas pLKO.pig was provided by I. Lemischka. Mxl-Cre and Flt $3^{I T D}$ mouse strains were kindly provided by G. Vassiliou. Vav-iCre mice were provided by P. Liu. We thank Louise Van Der Weyden and Anneliese Speak for help with mouse work. We are grateful to the Research Support and Cytometry Core Facilities at the Wellcome Sanger Institute for assistance in mouse husbandry and flow cytometry respectively. Primary human cells were provided by the Cambridge Blood and Stem Cell Biobank, which is supported by the Cambridge NIHR Biomedical Research Centre, Wellcome/MRC Stem Cell Institute and Cambridge Experimental Cancer Medicine Centre, UK. The Leucegene group is located at the Institute for Research in Immunology and Cancer in Montreal, Canada and is supported by Genome Canada and Genome Québec. J.-F.S. was supported by an CIHR fellowship.

\section{Author contributions}

E.S., S.R. and P.T. designed and performed experiments, analyzed data and reviewed the manuscript. V.I. K.W. and A.D. performed bioinformatic analysis and edited the manuscript. D.J.A contributed to study design, provided infrastructure support and edited the manuscript. J.-F.S. and G.S. were responsible for human survival analysis. C.C.W. conceived and directed the study, designed and executed experiments, analyzed data and wrote the manuscript.

\section{Competing interests}

The authors declare no competing interests.

\section{Additional information}

Supplementary information The online version contains supplementary material available at https://doi.org/10.1038/s41467-021-22750-8.

Correspondence and requests for materials should be addressed to C.C.W.

Peer review information Nature Communications thanks Robert Kralovics, Hermann Steller, Paresh Vyas and the other, anonymous, reviewer(s) for their contribution to the peer review of this work.

Reprints and permission information is available at http://www.nature.com/reprints

Publisher's note Springer Nature remains neutral with regard to jurisdictional claims in published maps and institutional affiliations.

\section{(c) (i)}

Open Access This article is licensed under a Creative Commons Attribution 4.0 International License, which permits use, sharing, adaptation, distribution and reproduction in any medium or format, as long as you give appropriate credit to the original author(s) and the source, provide a link to the Creative Commons license, and indicate if changes were made. The images or other third party material in this article are included in the article's Creative Commons license, unless indicated otherwise in a credit line to the material. If material is not included in the article's Creative Commons license and your intended use is not permitted by statutory regulation or exceeds the permitted use, you will need to obtain permission directly from the copyright holder. To view a copy of this license, visit http://creativecommons.org/ licenses/by/4.0/.

(C) The Author(s) 2021 